\title{
Title: Paradigm shift in eukaryotic biocrystallization
}

\author{
Authors: Jana Pilátová ${ }^{1,2,3}$, Tomáš Pánek ${ }^{4}$, Miroslav Oborník ${ }^{3,5}$, Ivan Čepička ${ }^{4}$, Peter Mojzeš²
}

\begin{abstract}
Affiliations:
${ }^{1}$ Department of Experimental Plant Biology, Faculty of Science, Charles University, Viničná 5, CZ-12844 Prague 2, Czech Republic

${ }^{2}$ Institute of Physics, Faculty of Mathematics and Physics, Charles University, Ke Karlovu 5, CZ12116 Prague 2, Czech Republic

${ }^{3}$ Institute of Parasitology, Czech Academy of Sciences, Branišovská 31, CZ-37005 České Budějovice, Czech Republic

${ }^{4}$ Department of Zoology, Faculty of Science, Charles University, Viničná 7, CZ-12844 Prague 2, Czech Republic

${ }^{5}$ Department of Molecular Biology and Genetics, Faculty of Science, University of South Bohemia, Branišovská 31, CZ-37005 České Budějovice, Czech Republic

*Correspondence to: jana.pilatova@natur.cuni.cz
\end{abstract}

Despite the widespread occurrence of crystalline inclusions in unicellular eukaryotes, scant attention has been paid to their composition, functions, and evolutionary origins, assuming just their inorganic contents. The advent of Raman microscopy, still scarcely used for biological samples, allowed chemical characterization of cellular inclusions in vivo. Using this method, herein we provide a substantial revision of the cellular crystalline inclusions across the broad diversity of eukaryotes examining all major supergroups. Surprisingly, here we show that $80 \%$ of these crystalline inclusions contain purines, mostly anhydrous guanine (62\%), guanine monohydrate (2\%), uric acid $(12 \%)$ and xanthine $(4 \%)$. Hence, our findings indicate that purine biocrystallization is a very general and an ancestral eukaryotic process operating by an as-yet-unknown mechanism. Purine crystalline inclusions are highcapacity and rapid-turnover reserves of nitrogen of a great metabolic importance, as well as optically active elements, e.g., present in the light sensing eyespots of flagellates, possessing even more hypothetical functions. Thus, we anticipate our work to be a starting point for more in-depth studies of this phenomenon on the detailed level spanning from cell biology to global ecology, with further potential applications in biotechnologies, bio-optics or in human medicine.

Crystalline inclusions, conspicuous in many single-celled eukaryotes, have attracted the attention of scientists since the emergence of microscopy. After Charles Darwin documented the crystallike particles in microscopic flagellates ${ }^{1}$, Ernst Haeckel coined the term "biocrystal" for calcite $\left(\mathrm{CaCO}_{3}\right)$, celestite $\left(\mathrm{SrSO}_{4}\right)$, silica $\left(\mathrm{SiO}_{2}\right)$, and oxalate in protists ${ }^{2}$. Biocrystals or biogenically originated crystalline inclusions of either organic or inorganic chemical nature, are typically formed inside vacuoles and grow into shells and scales on cell surfaces, e.g., calcite scales of coccolithophores that take part in the global carbon cycle ${ }^{3}$. On the other hand, rarely-reported 
purine inclusions of guanine, xanthine, hypoxanthine, or uric acid are often overlooked ${ }^{4-8}$. The recent resurgence of research on purine inclusions has shown rapid uptake kinetics of different nitrogen compounds that are converted into massive guanine nitrogen stores with sufficient capacity, in some cases, to support three consecutive cell cycles ${ }^{8}$. As a fundamental biogenic element, nitrogen represents a great share of biotic elemental stoichiometry, from cellular to global scales, impacting Earth's climate ${ }^{9}$. Biocrystalized guanine polarizes and reflects light in many animals, e.g., fish with the opalescent guanine crystals in the iridocytes of their scales resembling glittery camouflage, or as an adaptation for vision in scallops, deep-sea fishes and arthropods that exhibit guanine-reflective retinal tapeta in their eyes ${ }^{10,11}$. Analogous functions of guanine crystals in alveolate protists have also been reported ${ }^{5,12,13}$.

To date, the investigation of crystalline inclusions and their biological importance in unicellular eukaryotes has been impeded by a lack of methods for their non-destructive in situ and in vivo characterization. Although Raman microscopy ${ }^{8}$ provides just such capabilities (as detailed in Supplementary Materials and Methods), this methodology has not yet been fully exploited in the study of intracellular crystalline inclusions. Compared to other tools for microscopic elemental analysis (nanoSIMS, EELS, EDX TEM), or chemical analytical approaches (GC and LC MS, NMR, X-ray crystallography), Raman microscopy offers direct visualization of molecular profiles for various cellular compartments in single cells with the resolution of confocal microscopy and without the need for fixation, time-consuming and laborious sample preparation, or the need for large amounts of biological material necessary for chemical extractions. Thus, it is also suitable for environmental samples. Herein we take a major step forward in the research of crystalline cell inclusions by revisiting their chemical nature across the eukaryotic tree of life using Raman microscopy.

\section{Results}

We have screened all major eukaryotic groups, using $>200$ species, most of them for the first time, searching for birefringent (light-polarizing) crystalline inclusions. They are commonly located inside vacuoles, wobbling by Brownian motion (movies S1 to S8). Raman spectra of the biocrystals found are displayed in Figs. S1 to S5. We registered common biocrystals in accordance with previously-described crystalline inclusions, such as calcite, oxalate, celestite, and baryte ${ }^{3}$, together with other birefringent structures (i.e., starch, chrysolaminarin, strontianite, and newly observed crystals of sterols and carotenoids). However, apart from these, we found a surprisingly broad occurrence of purines ( $>80 \%$ of examined species containing crystals), particularly crystalline anhydrous guanine (62\%), uric acid $(12 \%)$, xanthine $(4 \%)$ and guanine monohydrate (2\%), (Fig. 1; Table S1; Supplementary Text). For the first time, we found anhydrous guanine purine crystal inclusions predominated in model and biotechnologically important species, and also in environmentally important strains. It commonly occurs in cosmopolitan marine and freshwater algae - including bloom-causing dinoflagellates, in the endosymbionts of corals crucial for the maintenance of entire coral reef ecosystems, in unicellular parasites of warmblooded animals and in cellulose-digesting anaerobic symbionts of termites, in slime molds etc. The lack of purine inclusions in some strains in our dataset (Table S1) does not exclude the existence of the necessary synthetic pathways since the induction of crystal formation may occur only under specific conditions.

We detected anhydrous guanine as the constituent of most purine crystals in all of the eukaryotic major groups (Fig. 1). Additionally, we found uric acid in cryptophytes, some diatoms, zygnematophytes, and klebsormidiophytes. We identified xanthine crystals in Amoebozoa, as well 
as in biotechnologically important microalgae (e.g., Chlorella and Isochrysis). Interestingly, we discovered the first occurrence of pure guanine monohydrate in marine diplonemids and as an admixture to uric acid in two green algae. Particular triggers for purine inclusion formation are unknown, but they are often produced after transfer to fresh growth media, media containing surplus sources of nitrogen, and under stress conditions ${ }^{4,6}$. As previously shown, purine crystals may act as nitrogen storage for microalgae in which they are formed in a type of luxury uptake resulting in net removal of nitrogen from the medium ${ }^{8}$.

Intracellular biocrystals, typically occurring inside membrane-bound compartments, are eukaryote-specific with the exception of bacterial magnetosomes ${ }^{3}$. The widespread distribution of purine crystals may be contingent on the emergence of cell compartmentalization in early eukaryotes. Furthermore, our results suggest that purine crystals might have been present in the last eukaryotic common ancestor (LECA), becoming one of the very first types of biocrystals in eukaryotes. Therefore, we employed comparative transcriptomics and genomics to identify candidate proteins that could be involved in the probably ancient pathways responsible for purine crystal formation. The components of the salvage pathway including phosphoribosyltransferases are such plausible candidate proteins. Consistent with this idea, we proved hypoxanthine-guanine phosphoribosyltransferase (HGPT) to be omnipresent among eukaryotes (Fig. S8 and S12) and we hypothesize that it might be responsible for purine reusage after crystal degradation and/or hydrolysis of nucleotides, releasing simple purines in order to form purine crystals. Furthermore, nucleobases (i.e., purines), nucleosides and/or nucleotides must be transported from cytoplasm to the vesicles where crystals are formed. Firstly, we focused on the most straightforward group of purine transporters that are considered to be commonly present among eukaryotes ${ }^{19}$. Our exhaustive homolog search and subsequent phylogenetic analyses surprisingly challenged the ubiquity of the three of them: nucleobase-cation symporter $1-\mathrm{NCS} 1$, nucleobase-ascorbate transporter - NAT, and AzgA (see Supplementary Text for details). Of these, only the AzgA transporter was probably present in LECA (see Fig. S8 and S9), although distribution of both its eukaryotic paralogs is rather limited. The evolutionary history of the other two known purine transporters in eukaryotes is much more complex and we do not have any strong evidence for their presence in LECA (Fig. S8, S10, and S11). NCS1 exists in eukaryotes in several paralogs, at least three of them emerged by relatively recent horizontal gene transfer from eubacteria (Fig. S10). NAT (= NCS2) emerged independently four times in eukaryotes (Fig. S11). Our final argument against involvement of solo-purine transporters in crystal formation is their absence in some of the purine crystal-forming groups (Heterolobosea, Ciliophora, and Apicomplexa). Next, we analyzed the distribution of nucleoside transporters, e.g., the concentrative (CNT) and equilibrative (ENT) nucleoside transporters, showing that CNT has an infrequent occurrence in eukaryotes (Table S2). The ENT family appears to be the most promising, as it is the only one among all nucleotide/nucleoside/nucleobase transporters we tested that has a clear pan-eukaryotic distribution (Table S3). Members of the ENT family are specific for nucleosides and nucleobases, and are part of the major facilitator superfamily (MFS). In general, ENTs can operate in a bidirectional mode, in some cases with cation symport and with different localization in the plasma membrane or in intracellular vesicles ${ }^{20}$. There are also other candidate carriers (e.g., VNUT that is known from metazoans), but the exact distribution, function, and localization of such proteins cannot be reliably predicted in silico without further biochemical studies in other eukaryotes ${ }^{21}$. The metabolism of nucleobases, nucleosides and nucleotides together with their transport is essential for all organisms and hence there may not be any purine transporters solely involved in 
crystal formation, as they likely play additional essential roles in the cell. Thus, extensive biochemical and proteomic studies have to be employed to answer this question in future.

\section{Discussion}

Intriguingly, the transition of crystal composition from purines in green algae to calcium oxalate in land plants may be metabolically bridged through purine degradation ${ }^{14,15}$. We also see a similar trend in Fungi, with yeasts possessing purine crystals, filamentous fungi producing calcium oxalate $^{7,16}$ and some marine seaweeds (Phaeophyceae) lacking crystals altogether. Hence, loss of the capacity to form purine crystals may correlate with the development of multicellularity that the necessity to store nitrogen is replaced by transfer of soluble metabolites through the multicellular body ${ }^{17}$. In some animals purine crystals, rather than serving as metabolic depots, instead function as photonic mirrors ${ }^{10,11}$. Others produce purines as a waste product mitigating the toxicity of excessive nitrogen uptake when excretion is not possible or is outstripped ${ }^{18}$. However, nitrogen is a growth-limiting factor for free-living microscopic eukaryotes ${ }^{9}$, and thus, a mechanism to store this essential element instead of wasting it is a significant advantage. Due to low-solubility and high-capacity, nitrogen-rich purine inclusions might have emerged as a preadaptation to nitrogen detoxification, protecting against exposure to high levels of ammonia or nitrates, utilizing vacuoles as a versatile sequestration space. On the other hand, in nitrogen depleted environments, nitrogen storage may mitigate stress-induced oxidative by allowing prompt production of glutathione, heatshock proteins, chaperones, peroxidases, etc.

Raising awareness of the existence of purine inclusions in diverse unicellular eukaryotes brings implications for other fields as well. The nitrogen-rich microbes might be of use in biofertilizers. The exceptional optical activity of purine crystals can be exploited in cosmetics for pearly iridescent effects or in optics for scalable surfaces with magnetically alterable reflectivity ${ }^{10}$. The value of algae-based food supplements may be limited by the medical issues associated with regular intake, of purines, e.g., hyperuricemia manifesting as gouty arthritis and increased cardiovascular risk, related to accelerated atherosclerosis ${ }^{22}$. Additionally, producers of algae-based biofuels and nutraceuticals commonly use nitrogen starvation to stimulate lipid production ${ }^{23}$. The implications of purine inclusions associated with this process have not yet been taken into account.

To understand the process of purine crystals formation and degradation may be crucial for treatments of hyperuricemia-caused urolithiasis or gout in human medicine. According to epidemiological evidence, the increasing prevalence of hyperuricemia $(\sim 21 \%)$ as one of the modern civilization diseases represents the main cause of gout ${ }^{22}$. Gout and uric acid nephropathy are caused by the purine crystals formation in joints and kidneys, respectively, and they are still of unknown mechanism. The current treatment is limited to xanthine oxidase inhibitors (e.g., allopurinol), as in most of the cases, there is no way to dissolve or remove the crystals already formed $^{22}$. In the course of evolution, humans might have lost the ability for purine crystals degradation. Thus, the understanding of purine biocrystallization in unicellular models can help to understand human pathophysiology and the molecular traits

of both: purine crystal formation in order to design specific inhibitors, as well as the mechanism of degradation in unicellular organism in order to introduce this system to the multicellular bodies for establishing the curative treatments of hyperuricemia-related diseases.

Purine inclusions, often overlooked but widely distributed in unicellular eukaryotes, serve as a high-capacity nitrogen storage mechanism and comprise a previously missing piece of the unsolved puzzle of the global nitrogen cycle. Indeed, most of the light-polarizing crystals present 
in various species of eukaryotes appear to contain purines, including guanine, uric acid and xanthine. This is in stark contrast to the traditional assumption that biocrystals are typically formed by the inorganic crystals, calcite and calcium oxalate ${ }^{2}$. The under-appreciated but widespread occurrence of purine inclusions, especially in unicellular eukaryotes, offers a rich source of material for future study in fields from cell biology to global ecology.

\section{References:}

1. Darwin, C. Journal of Researches Into the Geology and Natural History of the Varoius Countries Visited by HMS Beagle, Under the Command of Captain Fitzroy from 1832 to 1836 by Charles Darwin. Colburn (Colburn, 1840).

2. Haeckel, E. Kristallseelen: Studien über das anorganische Leben. (Alfred Kröner Verlag, 1917).

3. Raven, J. A. \& Knoll, A. H. Non-skeletal biomineralization by eukaryotes: Matters of moment and gravity. Geomicrobiol. J. 27, 572-584 (2010).

4. Creutz, C. E., Mohanty, S., Defalco, T. \& Kretsinger, R. H. Purine composition of crystalline cytoplasmic inclusions of Paramecium tetraurelia. Protist 153, 39-45 (2002).

5. Jantschke, A. et al. Anhydrous $\beta$-guanine crystals in a marine dinoflagellate: Structure and suggested function. J. Struct. Biol. 207, 12-20 (2019).

6. Moudř́k $\quad$ Sová, Š., Nedbal, L., Solovchenko, A. \& Mojzeš, P. Raman microscopy shows that nitrogen-rich cellular inclusions in microalgae are microcrystalline guanine. Algal Res. 23, 216-222 (2017).

7. Roush, A. H. Crystallization of purines in the vacuole of Candida utilis. Nature 190, 449 (1961).

8. Mojzeš, P. et al. Guanine, a high-capacity and rapid-turnover nitrogen reserve in microalgal cells. Proc. Natl. Acad. Sci. 117, 32722-32730 (2020).

9. Sterner, R. W. \& Elser, J. J. Ecological Stoichiometry: The Biology of Elements from Molecules to the Biosphere. Encyclopedia of Ecology, Five-Volume Set (Princeton University Press, 2002).

10. Tadepalli, S., Slocik, J. M., Gupta, M. K., Naik, R. R. \& Singamaneni, S. Bio-optics and bio-inspired optical materials. Chem. Rev. 117, 12705-12763 (2017).

11. Palmer, B. A. et al. The image-forming mirror in the eye of the scallop. Science (80-. ). 358, 1172-1175 (2017).

12. Kuhlmann, H. W., Bräucker, R. \& Schepers, A. G. Phototaxis in Porpostoma notatum, a marine scuticociliate with a composed crystalline organelle. Eur. J. Protistol. 33, 295-304 (1997).

13. Yamashita, H., Kobiyama, A. \& Koike, K. Do uric acid deposits in zooxanthellae function as eye-spots? PLoS One 4, 1-9 (2009).

14. Brychkova, G., Fluhr, R. \& Sagi, M. Formation of xanthine and the use of purine metabolites as a nitrogen source in Arabidopsis plants. Plant Signal. Behav. 3, 999-1001 (2008).

15. $\mathrm{Yu}, \mathrm{L}$. et al. Glyoxylate rather than ascorbate is an efficient precursor for oxalate biosynthesis in rice. J. Exp. Bot. 61, 1625-1634 (2010).

16. Gadd, G. M. et al. Oxalate production by fungi: Significance in geomycology, biodeterioration and bioremediation. Fungal Biol. Rev. 28, 36-55 (2014).

17. Winter, G., Todd, C. D., Trovato, M., Forlani, G. \& Funck, D. Physiological implications of arginine metabolism in plants. Front. Plant Sci. 6, 1-14 (2015). 
18. Wright, P. A. Nitrogen excretion: Three end products, many physiological roles. J. Exp. Biol. 198, 273-281 (1995).

19. Kourkoulou, A., Pittis, A. A. \& Diallinas, G. Evolution of substrate specificity in the nucleobase-ascorbate transporter (NAT) protein family. Microb. Cell 5, 280-292 (2018).

20. Boswell-Casteel, R. C. \& Hays, F. A. Equilibrative nucleoside transporters - a review. Nuclesides Nucleotides Nucleic Acids 36, 7-30 (2017).

21. Sawada, K. et al. Identification of a vesicular nucleotide transporter. Proc. Natl. Acad. Sci. U. S. A. 105, 5683-5686 (2008).

22. Bove, M., Cicero, A. F. G., Veronesi, M. \& Borghi, C. An evidence-based review on urate-lowering treatments: implications for optimal treatment of chronic hyperuricemia. Vasc. Health Risk Manag. 13, 23-28 (2017).

23. Ajjawi, I. et al. Lipid production in Nannochloropsis gaditana is doubled by decreasing expression of a single transcriptional regulator. Nat. Biotechnol. 35, 647-652 (2017). 

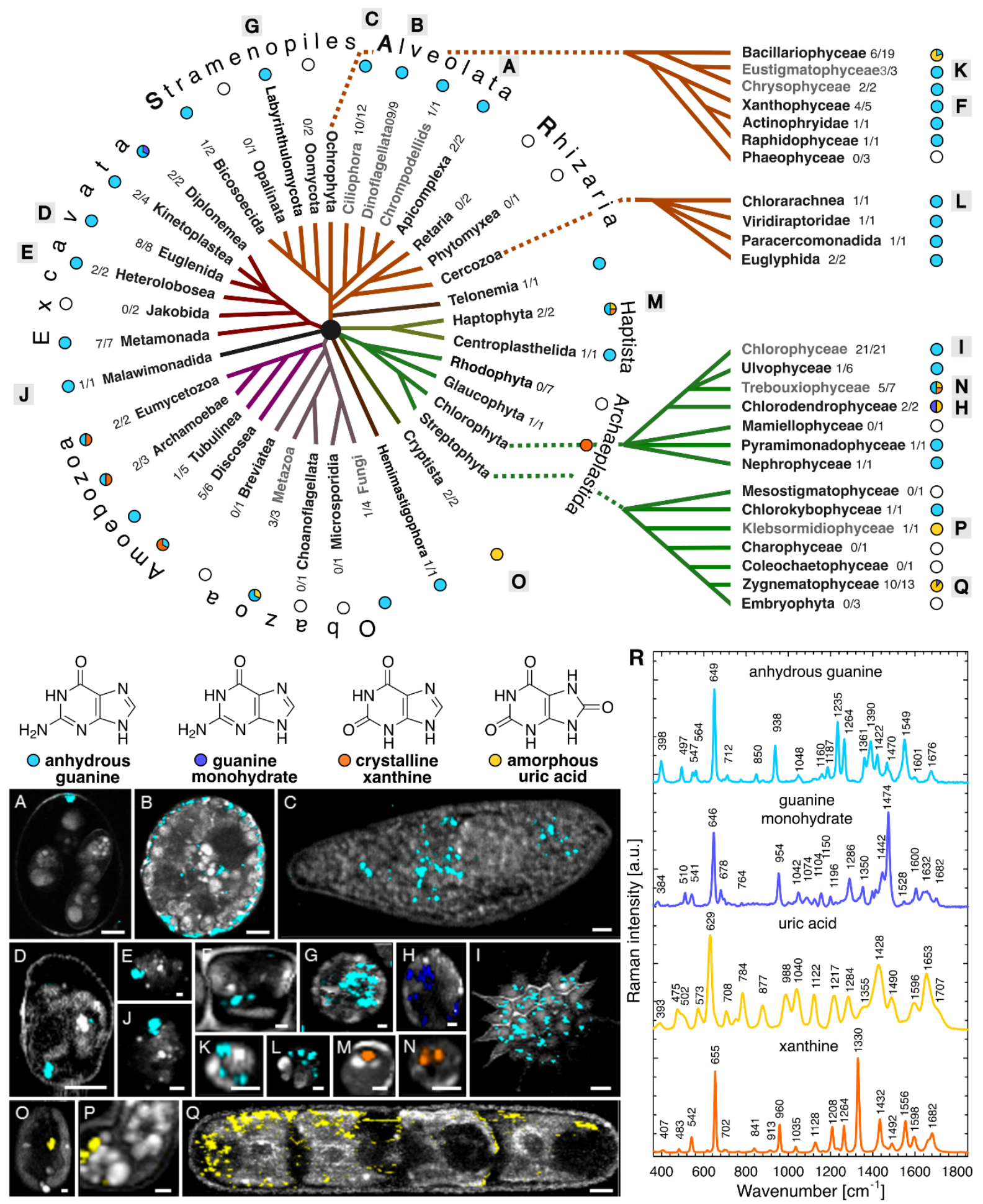

Fig. 1: Distribution of purine inclusions identified by Raman microscopy in the eukaryotic tree of life. The occurrence of anhydrous guanine (cyan), guanine monohydrate (violet), uric acid (yellow) and xanthine (orange) are illustrated in the evolutionary scheme as pie charts and in Raman maps (A-Q) together with the Raman spectra (R). Ratio of species positively tested for purine inclusions out of the total number of screened samples are expressed for each taxonomic category. Lineages highlighted in grey possess purine inclusions already reported elsewhere. A - 
bioRxiv preprint doi: https://doi.org/10.1101/2022.01.11.475817; this version posted January 11, 2022. The copyright holder for this preprint (which was not certified by peer review) is the author/funder. All rights reserved. No reuse allowed without permission.

Eimeria maxima, B - Glenodinium foliaceum, C - Paramecium sp., D - Eutreptiella gymnastica, E - Naegleria gruberi, F - Tribonema aequale, G - Schizochytrium sp., H - Tetraselmis subcordiformis, I - Pediastrum duplex, J - Gefionella okellyi, K - Nannochloropsis oculata, L Bigelowiella natans, $\mathrm{M}$ - Isochrysis sp., $\mathrm{N}$ - Chlorella vulgaris, O - Cryptomonas sp., $\mathrm{P}$ Klebsormidium flaccidum, Q - Penium margaritaceum Scale bars: $5 \mu \mathrm{m}$ (A-D, I, Q), $1 \mu \mathrm{m}$ (E-H). 


\section{Methods}

Biological material - cell cultures and environmental samples

To assess the composition of various birefringent cell inclusions among microscopic eukaryotes, we screened species from environmental samples, cell cultures obtained from various culture collections, and strains from private collections that were kindly donated by our collaborators (listed in Table S1 and acknowledgements). The cultivation conditions for each of the tested species are shown in Table S1. Environmental samples were assessed within one week after collection. Cell cultures were observed after transfer to fresh media on the same day and/or during five consecutive days until the purine inclusions were observed. In some cases (marked with "*" in Table S1), we transferred the cells to media containing dissolved guanine (approximately $30 \mu \mathrm{M}$ final concentration), in order to facilitate the formation of crystalline inclusions.

\section{Polarization microscopy}

We used polarization microscopy to screen organisms for intracellular crystalline inclusions. Initially, polarization microscopy was performed separately, using an Olympus AX 70 Provis microscope (Olympus, Japan) or confocal microscope (Leica TCS SP8; Leica, Germany) equipped with a digital camera (Leica MC170 HD; Leica, Germany). After installing the polarization filters directly on the Raman microscope (WITec alpha300 RSA, WITec, Germany), photomicrographs and videos were taken immediately before Raman measurements. Short videos were taken using the default settings (25 fps using the Leica TCS SP8 or 20 fps using the WITec alpha300 RSA). Videos were processed using Vegas Pro 14.0 software (MAGIX, Germany).

\section{$\underline{\text { Raman microscopy }}$}

Measurements and data-processing were performed as described elsewhere ${ }^{6,8,24}$. The advantages and possible drawbacks of this method have recently been discussed ${ }^{25}$. In brief, Raman map scanning of 203 species comprising more than 3000 measurements of whole cells, and/or single spectra of crystalline inclusions of mobile cells, or cells with fast-moving cytoplasm, , was done using a confocal Raman microscope (WITec alpha300 RSA) equipped with the following objectives: $20 \times$ EC Epiplan, NA $=0.4$ (Zeiss, Germany), 50 $\times$ EC Epiplan-Neofluar, NA $=0.55$ (Zeiss, Germany), 60× water-immersion UPlanSApo, NA 1.2 (Olympus, Japan), 100× oilimmersion UPlanFLN, NA 1.3 (Olympus, Japan). A $532 \mathrm{~nm}$ laser with a power of approximately $20 \mathrm{~mW}$ at the focal plane was used.

For Raman map scanning, cell cultures were used as follows: $1 \mathrm{ml}$ of culture was centrifuged at $2000 \mathrm{~g}$ for $1 \mathrm{~min}$, when necessary for fast-moving flagellates, immobilization was accomplished by mixing $5 \mu \mathrm{l}$ of the cell pellet with $5 \mu \mathrm{l}$ of $1 \%$ low-temperature-melting agarose spread under a $20 \mathrm{~mm}$ diameter, $0.18 \mathrm{~mm}$-thick quartz coverslip sealed with CoverGrip (Biotium, USA). In cases of environmental samples, where assessment of cell movement is crucial for reliable identification of species (mostly Amoebozoa and Excavata), immobilization was not used, and data acquisition was performed via Raman single-spectrum mode with an integration time of $0.5 \mathrm{~s}$ and 20 accumulations using one of the following objectives: $50 \times$ EC Epiplan- Neofluar, $60 \times$ UPlanSApo, and 100× UPlanFLN. Raman map measurements were performed with a scanning step of $200 \mathrm{~nm}$ in the both directions, voxel size $1 \mu^{3}$ and an integration time of $0.07 \mathrm{~s}$ per voxel with either the $60 \times$ UPlanSApo or $100 \times$ UPlanFLN objectives. On average, we measured 3-10 cells of each strain from each cell culture. In case of environmental samples, we measured at least 
one cell. Standards of pure chemical substances were measured in water suspension. To prepare the matching references for biogenic crystals of uric acid, guanine monohydrate, xanthine, and their mixtures, the substances were dissolved in an aqueous solution (4\%) of dimethylamine (DMA) and dried on the quartz slide to allow recrystallization.

Data was analyzed using WITec Project FIVE Plus v5.1 software (WITec, Germany) to implement the following steps: cosmic ray removal, background subtraction, cropping of the spectral edges affected by detector margins, spectral unmixing with the true component analysis tool, and averaging of the mean spectrum, summarizing multiple measurements in order to optimize the signal-to-noise ratio for each single spectrum of the crystalline inclusions.

\section{Phylogenetic analyses}

In an attempt to evaluate the role of nucleobase-cation symporter 1 (NCS1), nucleobaseascorbate transporter (NAT), AzgA, and hypoxanthine-guanine phosphoribosyl transferase (HGPT) in purine crystal biocrystallization, we tested their phylogenetic distribution and the robustness of the phylogenetic placements using methods of molecular phylogenetics. We performed an extensive set of searches of eukaryotic and prokaryotic sequence databases. Using several representative sequences of each gene as queries, we performed a BLASTp search against 87 high-quality, well-annotated eukaryotic and prokaryotic genomes and transcriptomes. To exclude the possibility that absence of NCS1, NAT, and AzgA gene in predicted proteomes from Heterolobosea, Ciliophora, and Apicomplexa is caused by suboptimal protein prediction, we also checked the presence of their homologs (using TBLASTN search) in contigs from eight nucleotide genome assemblies representing the three groups. While HGPT homologs were easy to detect, TBLASTN did not detect any purine transporter genes. Thus, we can be confident that the absence of these genes in genomic data is not artificial. Datasets containing original sequences and their manually curated homologs, identified by BLASTp search, were included into initial datasets and aligned by MAFFT version 7. These alignments were used as inputs to build Hidden Markov Models for a final sensitive homolog search by HMMER3 software ${ }^{26}$ to identify candidate proteins from 742 eukaryotic genomes and transcriptomes included in the EukProt database of genomescale predicted proteins across the diversity of eukaryotes ${ }^{27}$. To avoid bias introduced by contaminations or erroneous protein predictions in the EukProt database, we performed a preliminary set of Maximum-Likelihood phylogenetic analyses using IQ-TREE multicore version 1.6.10 ${ }^{28}$ under LG4X model. Between every round, we manually inspected each tree to identify possible eukaryotic and prokaryotic contaminations. Suspicious sequences were used as queries against the NCBI database of non-redundant proteins (nr) and best blast hits were added to the dataset. Final gene datasets, free of contaminant sequences and in-paralogs (recent gene duplications that resulted in several homologs with almost identical sequence), were aligned by MAFFT ${ }^{29}$. Alignments were manually edited in BioEdit ${ }^{30}$, phylogenetic trees were constructed by the maximum likelihood method using RAxML ${ }^{31}$, with $\mathrm{LG}+\mathrm{GAMMA}+\mathrm{F}$ model selected by Modelgenerator $^{32}$, and 200 nonparametric bootstrap analyses. Trimmed datasets of AzgA, NCS1, NCS2, NATs, and HGPRT protein families are stored on an online depository server, which will be accessible upon full article publication: https://figshare.com/s/ec36ff8263c1114d547a.

For assessing the distribution of equilibrative nucleoside transporter or solute carrier 29 (ENT, SLC29) and concentrative nucleoside transporter (CNT, SLC28), we used seed-sequences according to the references ${ }^{33}$ as initial datasets aligned by MAFFT version 7 . These alignments 
were used as inputs to build a profile HMM followed by an HMM search against 57 sequences of genomes using HMMER3 software ${ }^{26}$.

Data and materials availability: All data is available in the main text or the supplementary materials.

\section{Methods references:}

24. Barcytè, D., Pilátová, J., Mojzeš, P. \& Nedbalová, L. The arctic Cylindrocystis (Zygnematophyceae, Streptophyta) green algae are genetically and morphologically diverse and exhibit effective accumulation of polyphosphate. J. Phycol. 56, 217-232 (2020).

25. Moudř́ḱková, Š. et al. Comparing biochemical and Raman microscopy analyses of starch, lipids, polyphosphate, and guanine pools during the cell cycle of Desmodesmus quadricauda. Cells 10, 1-21 (2021).

26. Eddy, S. R. A probabilistic model of local sequence alignment that simplifies statistical significance estimation. PLoS Comput. Biol. 4, 1-14 (2008).

27. Richter, D. J., Berney, C., Strassert, J. F. H., Burki, F. \& de Vargas, C. EukProt: A database of genome-scale predicted proteins across the diversity of eukaryotic life. bioRxiv 1-11 (2020).

28. Hoang, D. T., Chernomor, O., Von Haeseler, A., Minh, B. Q. \& Vinh, L. S. UFBoot2: Improving the ultrafast bootstrap approximation. Mol. Biol. Evol. 35, 518-522 (2018).

29. Katoh, K. \& Standley, D. M. MAFFT multiple sequence alignment software version 7: Improvements in performance and usability. Mol. Biol. Evol. 30, 772-780 (2013).

30. Hall, T. BioEdit: a user-friendly biological sequence alignment editor and analysis program for Windows 95/98/NT. Nucleic Acids Symp. Ser. 41, 95-98 (1999).

31. Stamatakis, A. RAxML version 8: A tool for phylogenetic analysis and post-analysis of large phylogenies. Bioinformatics 30, 1312-1313 (2014).

32. Keane, T. M., Creevey, C. J., Pentony, M. M., Naughton, T. J. \& McInerney, J. O. Assessment of methods for amino acid matrix selection and their use on empirical data shows that ad hoc assumptions for choice of matrix are not justified. BMC Evol. Biol. 6, 1-17 (2006).

33. Young, J. D., Yao, S. Y. M., Baldwin, J. M., Cass, C. E. \& Baldwin, S. A. The human concentrative and equilibrative nucleoside transporter families, SLC28 and SLC29. Mol. Aspects Med. 34, 529-547 (2013). 
Acknowledgments: We express our gratitude to Kateřina Schwartzerová, Jan Petrášek, Stanislav Vosolsobě, Lukáš Falteisek, Jana Krtková and Richard Dorrell for constructive discussions. English has been kindly corrected by William Bourland. Furthermore, we thank to Dovilè Barcytè, William Bourland, Antonio Calado, Dora Čertnerová, Yana Eglit, Ivan Fiala, Martina Hálová, Miroslav Hyliš, Dagmar Jirsová, Petr Kaštánek, Viktorie Kolátková, Alena Kubátová, Alexander Kudryavtsev, Frederik Leliaert, Julius Lukeš, Jan Mach, Joost Mansour, Jan Mourek, Yvonne Němcová, Fabrice Not, Vladimír Scholtz, Alastair Simpson, Pavel Škaloud, Jan Št’astný, Róbert Šut'ák, Daria Tashyreva, Dana Savická, Jan Šobotník, Zdeněk Verner, Jan Votýpka for kindly providing cultures and taxonomic identifications. Funding: Financial support from the Czech Science Foundation (grants 17-06264S, 19-19297S, 20-16549Y, 21-03224S, and 21-26115S); Grant Agency of Charles University (grant 796217), Charles University Research Centre program No. 204069, European Regional Development Fund and the state budget of the Czech Republic, projects no. CZ.1.05/4.1.00/16.0340, CZ.1.05/4.1.00/16.0347, CZ.2.16/3.1.00/21515 and CZ.02.1.01/16_019/0000759,_LM2018129.

Author contributions: JP conceived the study, handled the cell cultures, performed the Raman measurements and data processing, prepared the graphics and videos and wrote the manuscript; TP and MO performed phylogenetic analyses and profiling; PM conceived the study, processed the data and corrected the manuscript; IC provided the cell cultures and corrected the manuscript. All authors discussed and approved the manuscript.

Competing interest declaration: Authors declare no competing interests. 
Supplementary Information is available for this paper.

\section{Supplementary Text}

\section{Results}

Here we provide a detailed description of the results presented in Fig. 1 and Table S1. We examined representatives of all currently recognized eukaryotic supergroups ${ }^{34,35}$ for the presence and composition of purine inclusions, i.e., guanine anhydride, guanine monohydrate, uric acid and xanthine. To the best of our knowledge, this is the first report on the occurrence of pure crystalline guanine monohydrate in any microorganism. Apart from purine inclusions being formed by pure substances (Fig. S1), we also found four species forming mixed crystals consisting of various proportions of guanine monohydrate, uric acid and/or xanthine (Fig. S2).

Guanine inclusions (Movie S1) were ubiquitous within the SAR clade (Stramenopiles, Alveolata, Rhizaria). In Alveolata, we confirmed, for the first time, that the morphologically prominent "polar granules" in unsporulated oocysts of the parasitic apicomplexan Eimeria maxima consist of guanine. Furthermore, we proved that inclusions in all observed alveolates, including parasitic apicomplexans (Eimeria maxima, Psychodiella sergenti), photoparasitic chromerids ${ }^{36}$, and very diversified and ecologically important dinoflagellates and ciliates consist exclusively of guanine. Among dinoflagellates, our sampling included clades with species having plastids derived from diatoms (Glenodinium foliaceum) and also those having complex plastids of rhodophyte origin ${ }^{37}$. Moreover, we also included the bloom- or red-tide-causing species Heterocapsa triquetra 38 and a fresh isolate of Symbiodiniaceae from the soft coral Capnella imbricata.

Compared to Alveolata, in Stramonopiles the situation is more complex. Guanine crystals predominate in most species, such as predatory actinophryids, bicosoecids, phototrophic and heterotrophic chrysophytes (Synura hibernica, Spumella sp., respectively), biotechnologically promising eustigmatophytes (Nannochloropsis oculata, Eustigmatos cf. polyphem) ${ }^{39,40}$, labyrinthulomycetes (Schizochytrium sp.) ${ }^{41}$, raphidophytes (Gonyostomum sp.), and xanthophytes (Botrydiopsis intercedens, Tribonema aequale, Xanthonema sp.). In contrast, diatoms have a lower prevalence of crystalline inclusions than other stramenopiles and exhibit production of uric acid crystals, for example freshwater species of Encyonema, Fragilaria, Navicula, and Pleurosigma. Guanine crystals were detected in only a single marine/brackish diatom species (Naviculaceae gen. sp., Seminavis-like). Diatoms are known for a very complex nitrogen metabolism employing a urea cycle comparable to the one in animals ${ }^{42}$. Crystalline inclusions were not detected in the parasitic Blastocystis (Opalinata) or in Oomycota. Guanine crystals predominated in the sampled Rhizaria (i.e., Cercozoa). In Gromiida and in Retaria (Foraminifera, Acantharea, Polycystinea) we suspected the previously described structures called "stercomata" ${ }^{43}$ to be crystals of purines, calcite or celestite (strontium sulfate). For now, we did not prove any of those with certainty due to low sampling of only three species of formalin-fixed cells of Acantharea and Foraminifera.

The more intricate crystals of Haptista may contain a mixture of both purines (Movie S2). Among haptophytes, Emiliania huxleyi ${ }^{44}$, a model organism with ecological importance, possesses guanine crystals, whereas the biotechnologically important Isochrysis sp. ${ }^{41}$ has xanthine crystals. In both cases, organisms were cultured in guanine-supplemented medium (Table 1). Centroplasthelida from freshwater habitats (Rhaphidiophrys sp.) possessed numerous guanine crystals. Telonemida (Telonema sp.) contained guanine inclusions when cultured in guaninesupplemented medium. Within Cryptista (Chroomonas sp., Cryptomonas sp.) we showed that the highly refractile and taxonomically important Maupas body ${ }^{45}$ consists of uric acid. 
In Archaeplastida (Movie S3), guanine crystals were present in glaucophytes but were not detected in any of the nine sampled rhodophyte species. Guanine inclusions are consistently distributed throughout the UTC clade (Ulvophyceae, Chlorophyceae, and Trebouxiophyceae) except for the biotechnologically significant species, Chlorella vulgaris ${ }^{41}$, which possess xanthine, and Dictyosphaerium sp. ${ }^{46}$, which contains uric acid crystals. The arctic species Chloromonas arctica ${ }^{47}$ possesses guanine crystals. Interestingly, Chlamydomonas in culture or environmental samples possesses guanine crystals in different life stages from flagellate to palmelloid. Xanthine is also present in the crystals of chlorodendrophytes, including another biotechnologically exploited species, Tetraselmis subcordiformis ${ }^{41}$, whereas other marine chlorophyte counterparts contain guanine crystals (e.g. Nephroselmis sp.). The smallest free-living eukaryote, with the size of bacteria, Ostreococcus tauri (Mamiellophyceae) ${ }^{48}$, did not show any crystalline inclusions even though they may contain starch as a storage polysaccharide. It is also possible that our methods had insufficient resolution to detect crystalline inclusions in these tiny cells. In stark contrast to Chlorophyta, the Streptophyta ${ }^{49}$, notably including land plants (Embryophyta) together with the related microalgae, Zygnematophyceae and Klebsormidiophyceae, contain uric acid inclusions. In the case of Mesotaenium caldariorum, the predominant uric acid include admixtures of crystalline guanine monohydrate. However, other examined streptophytes such as Chlorokybus atmophyticus (Chlorokybophyceae) form guanine crystals. We detected no crystalline inclusions in Mesostigma viride (Mesostigmatophyceae). In Embryophyta, Coleochaetophyceae, and Zygnematophyceae we observed calcium oxalate inclusions instead of purine crystals (see details below).

Crystalline inclusions of varied chemical composition, primarily guanine and xanthine, occur commonly in Amoebozoa (Movie S4). Xanthine crystals, uncommon in other eukaryotes, are present in freshwater and marine Mayorella sp., in the facultatively parasitic model organism, Acanthamoeba castellanii, in the anaerobic model species Mastigamoeba balamuthi, and a model terrestrial slime mold, Physarum polycephalum ${ }^{50,51}$. By contrast, guanine was present in a fresh isolate of another slime mold, Fuligo septica, a different species of Archamoebae (Mastigella eilhardi), and in species of the common genera Thecamoeba, Vannella, and Difflugia.

We showed by Raman microscopy that, in Opisthokonta, uric acid, rather than guanine, is a common excretory product of nitrogen metabolism (e.g. in nematodes). Interestingly, we noticed guanine microcrystals inside swarming acoelomate gastrotrichs (Platyzoa) in a sample from a peat bog (Movie S5). Using Raman microscopy, we also confirmed that guanine crystals serve as a refractile layer on fish scales ${ }^{52}$. In Fungi (Holomycota) we found crystalline guanine in Candida albicans, which has been tested for uptake of different purine compounds previously ${ }^{53}$. However, we did not find any crystalline inclusions in Saccharomyces cerevisiae. We observed no crystalline inclusions in either intracellular parasites belonging to Microsporidia or in the freeliving halophilic Choanoflagellata. We did not detect crystalline inclusions in the Breviatea, close relatives of opisthokonts.

In Excavata (Movie S6), the crystalline inclusions, present in all studied lineages, were exclusively composed of guanine, including: both heterotrophic (Entosiphon sp., Rhabdomonas sp.) and photosynthetic euglenids, freshwater and marine euglenids (Euglena sp., Eutreptiella gymnastica, respectively), in free-living kinetoplastids (but not in the parasites, such as trypanosomatids), in deep-sea diplonemids (Namystinia karyoxenos, Rhynchopus sp.) ${ }^{54,55}$, aerotolerant heteroloboseans (Naegleria gruberi) ${ }^{56}$, and strictly anaerobic metamonad symbionts of termites (e.g. Macrotrichomonoides sp. isolated from Neotermes cubanus) ${ }^{57}$. In the diplonemid, Flectonema sp., guanine monohydrate was detected in the form of pure crystals, probably 
monocrystals, as their Raman spectra exhibit similar variability of relative intensities when excited by polarized light to that of synthetically prepared monocrystals.

We found guanine crystals in Gefionella okellyi, from the phylogenetically distinct clade of Malawimonadida ${ }^{58}$. Among the CRuMs group (Collodictyonida, Rigifilida, and Mantamonadida) ${ }^{59}$, we tested mantamonads but found no crystalline inclusions inside their cells, even after exposing them to guanine-enriched culture medium. Lastly, crystalline guanine was found in Hemimastix kukwesjijk, the only representative sampled from Hemimastigophora ${ }^{58}$, a separate, deep-branching lineage, recently described as a new eukaryotic supergroup (Movie S7).

In addition to the predominant purine crystalline inclusions $(80 \%)$, there are also other types. Surprisingly, calcite (calcium carbonate $-\mathrm{CaCO}_{3}$ ) was present in diatoms ${ }^{60}$, in which amorphous silica (silicon dioxide - $\mathrm{SiO}_{2}$ ), that does not polarize light, is the main component of their biomineralized frustules ${ }^{61}$. Calcification (Fig. S3, Tab. S1) occurred in the seaweeds tested, including rhodophytes, ulvophytes and phaeophytes, indicating that this process occurs very commonly in marine environments ${ }^{62}$. Similarly, calcified shells of foramiferans and the calcite scales on the cell surface of the haptophytic coccolithophore Emiliania huxleyi also strongly polarized light ${ }^{63}$. Massive calcite incrustations occurred on surface of filamentous algae, e.g. Oedogonium sp., that has been previously described as crystal jewels in other freshwater filamentous algae ${ }^{64}$. We confirmed strontianite (strontium carbonate $-\mathrm{SrCO}_{3}$ ) in the green alga Tetraselmis, previously reported elsewhere using different methodology ${ }^{65}$.

Crystalline sulfates occurred in various species but only rarely (Fig. S4). Similarly, in the light-polarizing armor of Acantharea, we confirmed the presence of celestite (strontium sulfate $\left.\mathrm{SrSO}_{4}\right)^{66,67}$. Interestingly, we found baryte (barium sulfate $-\mathrm{BaSO}_{4}$ ) in three species of zygnematophytes (Closterium peracerosum-strigosum-littorale complex, Cosmarium sp., and Spirogyra sp.), for two of which it has been previously reported ${ }^{68,69}$. In laboratory cultures and environmental isolates of Saccamoeba sp. we found an unprecedently complex spectrum corresponding to numerous crystalline inclusions lacking light-polarization features. The dominating peak at $998 \mathrm{~cm}^{-1}$ (Fig. S4, Tab. S1) resembles either a signal of sulfates or aromatic compounds. The rest of the spectrum also shows lipid-like organic matter, thus, the crystals may be formed by a complex mixture of lipids and sulfates.

Calcium oxalate monohydrate $\left(\mathrm{CaC}_{2} \mathrm{O}_{4} \cdot \mathrm{H}_{2} \mathrm{O}\right)$ crystals were restricted to closely related streptophytic algae and land plants: Coleochaetophyceae, Zygnematophyceae (Cylindrocystis sp.), and Embryophyta (e.g. plant models Physcomitrella patens, Nicotiana tabacum) together with calcium oxalate dihydrate $\left(\mathrm{CaC}_{2} \mathrm{O}_{4} \cdot 2 \mathrm{H}_{2} \mathrm{O}\right)$ commonly found in Embryophyta (Fig. S5, Tab. S1). In the Embryophyta, deposition of calcium oxalate is already known to occur under stress conditions ${ }^{70}$.

Unexpected light-polarizing lipophilic inclusions unreported until now, occurred in some of examined samples. Compared to carotene crystals observed in the model plant Arabidopsis thaliana ${ }^{71}$, we found possibly similar structures in aerophytic ulvophytes (Trentepohlia sp., Scotinosphaera gibberosa) and freshwater cyanobacteria (Oscillatoria sp.), comprising a mixture of carotenoids and lipids containing sterols and fatty acids (Fig. S6, Table S1, Movie S8). Similarly, in the green parasitic alga, Phyllosiphon arisari ${ }^{72}$ and a symbiont of lichens, Symbiochloris tschermakiae ${ }^{73}$, their light-polarizing lipophilic crystals were a complex mixture of lipids, with a high proportion of unsaturated fatty acids in the former, and saturated fatty acids in the latter. Faintly light-polarizing lipophilic structures in amoebozoans (Entamoeba histolytica) resembled those mentioned above or contained a surplus of sterol compounds (Acanthamoeba castellanii and Mastigamoeba balamuthi). Some of them may be of a crystalline nature but this 
requires further evidence. All lipophilic light-polarizing structures tended to melt under prolonged illumination by a focused laser beam (ca $20 \mathrm{~mW}$ power) during the Raman measurements.

Refractile structures such as storage polysaccharides, i.e., starch or chrysolaminarin ${ }^{74,75}$, or aerotopes, air-filled vesicles with a reflective interface inside the cells of cyanobacteria ${ }^{76}$, might be confused with birefringent crystals. High-intensity light-polarization also occurs in the thick cellulose cell walls of ulvophytes, zygnematophytes, rhodophytes, glaucophytes and others, a phenomenon best-studied in plants ${ }^{77}$, or in starch, a storage polysaccharide of Archaeplastida and in chrysolaminarin, storage polysaccharide of SAR (Fig. S7, Table S1).

\section{Comments on Raman spectra analysis}

In some protists, a monohydrate of guanine in crystalline form was detected (Figs. S1 and S2). To the best of our knowledge, this is the first report confirming the occurrence of crystalline guanine monohydrate in any microorganism. To date, only a $\beta$-polymorph of crystalline guanine anhydride was detected in various organisms, including some microalgae and protists $4,5,8$. In Flectonema sp., guanine monohydrate was detected in the form of pure crystals (Fig. S1), probably monocrystals. We observed a few inclusions formed by purine mixtures, which has also been reported from Paramecium ${ }^{4}$. In the case of Mesotaenium caldariorum, crystalline guanine monohydrate seems to be only a minor admixture in the more abundant uric acid (Fig. S2), however its presence was demonstrated by spectral similarity with synthetically prepared samples containing both uric acid and guanine monohydrate. The inverse proportion of the two compounds has been found in Tetraselmis subcordiformis. Isochrysis sp. and Chroomonas sp. exhibited crystalline mixtures of xanthine and guanine monohydrate.

\section{Comments on phylogenetic analyses}

The nucleobase cation symporter-1 (NCS1) family (Fig. S10) of secondary active transport proteins includes proteins from prokaryotes and several lineages of eukaryotes. We recovered NCS1 eukaryotic paralogs - fungal Fcy type, fungal Fur type, algal type, and plant type as previously described ${ }^{78}$. Besides, we also identified four as-yet-unknown eukaryotic paralogs that we marked as NCS1 A-D. Distribution of NCS1 in eukaryotes is extremely patchy as summarized on Fig. S8. We conclude that NCS1 transporter has been acquired by eukaryotes several times independently. Fungal Fcy type is present in various Fungi (Ascomycetes, Basidiomycetes, Gonapodya). Some Fungi even contain several distant paralogs of this gene (e.g. Aspergillus and Candida). Interestingly, homologs from Oomycota form a clade within fungal sequences, branching sister to Aspergillus sequences with full statistical support. Thus, our analysis strongly indicates lateral transfer of this gene from Fungi (supergroup Obazoa) to Oomycota (supergroup SAR). We identified fungal Fcy type of NCS1 transporter also in Ktedonobacteria (Chloroflexi) and two groups of Proteobacteria (Betaproteobacteria and Gammaproteobacteria). Bacterial homologs of fungal Fcy form a clade branching within fungal sequences but, in this case, with relatively low bootstrap support. Besides, the whole clade of fungal Fcy sequences shows strong affinity to eubacterial permeases including those from Gammaproteobacteria and Betaproteobacteria, so in this case, lateral transfer of Fcy from Fungi to Eubacteria is less convincing. We also identified an ecological relationship between eukaryotes that possess fungal Fcy gene, all of which are adapted to extract nutrients from plants. The Fur type of NCS1 is present exclusively in Basidiomycetes and Ascomycetes (Fungi). The source organism for Fur type is unclear. The algal type of NCS1 is present in Nanochloropsis (SAR), Rhodophyta, and Chlorophyta (Archaeplastida). It also has an uncertain origin. Plant type NCS1 
was detected in Chlorophyta and Streptophyta (closely related lineages of the supergroup Archaeplastida). Surprisingly, this gene is also present in Rhodelphidia, another deep-branching lineage of the supergroup Archaeplastida. The gene has unclear origin, although it shows affinity to Proteobacteria with low statistical support.

We also identified four novel clades of eukaryotic NCS1 transporters. One of them is present only in dinoflagellates and shows a close relationship to cytosine permease from Actinobacteria (with full bootstrap support); another is in Chromera, diatoms, and dinoflagellates; it shows affinity to Bacteroidetes and Planctomycetes with full bootstrap support. The other clade contains homologs from choanoflagellates, Hemimastigophora, telonemids, dinoflagellates, Chlorophyta, and malawimonadids. The phylogenetic position of Incisomonas marina (Stramenopiles) within choanoflagellates is probably due to contamination, also most likely in the case of Paulinella (Rhizaria) within Chlorophyta. Finally, two sequences from unrelated amoebozoans (Filamoeba and Vermamoeba) form a robust clade with no affinity to any other group, forming another eukaryotic NCS1 clade.

The AzgA gene encodes a hypoxanthine-adenine-guanine transporter (Fig. S9) that is present in all main groups of eukaryotes except supergroup Amoebozoa, and it is also missing in metazoans. Our analysis convincingly shows that eukaryotic AzgA has a single origin in eukaryotes and has been probably present in two paralogs in the last eukaryotic common ancestor. We named those paralogs AzgA A and B. Besides, the analysis indicates a series of gene duplications during evolution of certain groups as seen in Chlorophyta and Dinoflagellata.

Nucleobase-Ascorbate Transporter (NAT) protein family (Fig. S11) is an extensively studied group of proteins. All bacterial NATs are $\mathrm{H}^{+}$symporters highly specific for either uracil or xanthine or uric acid. The fungal and plant members are $\mathrm{H}^{+}$symporters specific either for xanthine-uric acid, or for adenine-guanine-hypoxanthine-uracil. In contrast to the microbial and plant proteins, most functionally characterized mammalian NATs are highly specific for Lascorbate $/ \mathrm{Na}^{+}$. However, the rSNBT1 NAT transporter from a rat is specific for nucleobases ${ }^{19}$. Our analysis convincingly shows that NAT proteins were introduced to eukaryotes at least four times independently (NAT A-D) and have their closest homologs in eubacteria, NAT B and D with high statistical support. Metazoan and plant NATs both belong to the NAT A clade that is, together with NAT C, the most widespread NAT gene in eukaryotes. In contrast, NAT B is present only in Fungi and dictyostelids; NAD D is unique for Tritrichomonas and it was established by horizontal gene transfer from Firmicutes (it is encoded on tritrichomonas-like genomic contig, so it is not contamination). Interestingly, some well-supported eukaryotic clades are not congruent with eukaryotic phylogeny confounding interpretation of the descent of this protein family. In some cases, it might be explained by eukaryote-to-eukaryote horizontal gene transfers (e.g., from fungi to dictyostelids in NAT B).

Hypoxanthine-guanine phosphoribosyl transferase (HGPT) (Fig. S12), omnipresent in eukaryotes, can also be found in Eubacteria, Archaea and, surprisingly enough, we detected HGPT homologs also in Nucleocytoviricota genomes. Because it is a relatively short and divergent protein, we were unable to resolve its detailed phylogeny. Based on previously introduced nomenclature ${ }^{79}$, we distinguished a clade of "fungal HGPT" which is very divergent from the other "classical HGPTs". However, we were able to find homologs of "fungal HGPT" in virtually all eukaryotic supergroups.

\section{Supplementary references:}

1. Darwin, C. Journal of Researches Into the Geology and Natural History of the Varoius 
Countries Visited by HMS Beagle, Under the Command of Captain Fitzroy from 1832 to 1836 by Charles Darwin. Colburn (Colburn, 1840).

2. Haeckel, E. Kristallseelen: Studien über das anorganische Leben. (Alfred Kröner Verlag, 1917).

3. Raven, J. A. \& Knoll, A. H. Non-skeletal biomineralization by eukaryotes: Matters of moment and gravity. Geomicrobiol. J. 27, 572-584 (2010).

4. Creutz, C. E., Mohanty, S., Defalco, T. \& Kretsinger, R. H. Purine composition of crystalline cytoplasmic inclusions of Paramecium tetraurelia. Protist 153, 39-45 (2002).

5. Jantschke, A. et al. Anhydrous $\beta$-guanine crystals in a marine dinoflagellate: Structure and suggested function. J. Struct. Biol. 207, 12-20 (2019).

6. Moudř́k $\quad$ Sová, Š., Nedbal, L., Solovchenko, A. \& Mojzeš, P. Raman microscopy shows that nitrogen-rich cellular inclusions in microalgae are microcrystalline guanine. Algal Res. 23, 216-222 (2017).

7. Roush, A. H. Crystallization of purines in the vacuole of Candida utilis. Nature 190, 449 (1961).

8. Mojzeš, P. et al. Guanine, a high-capacity and rapid-turnover nitrogen reserve in microalgal cells. Proc. Natl. Acad. Sci. 117, 32722-32730 (2020).

9. Sterner, R. W. \& Elser, J. J. Ecological Stoichiometry: The Biology of Elements from Molecules to the Biosphere. Encyclopedia of Ecology, Five-Volume Set (Princeton University Press, 2002).

10. Tadepalli, S., Slocik, J. M., Gupta, M. K., Naik, R. R. \& Singamaneni, S. Bio-optics and bio-inspired optical materials. Chem. Rev. 117, 12705-12763 (2017).

11. Palmer, B. A. et al. The image-forming mirror in the eye of the scallop. Science (80-. ). 358, 1172-1175 (2017).

12. Kuhlmann, H. W., Bräucker, R. \& Schepers, A. G. Phototaxis in Porpostoma notatum, a marine scuticociliate with a composed crystalline organelle. Eur. J. Protistol. 33, 295-304 (1997).

13. Yamashita, H., Kobiyama, A. \& Koike, K. Do uric acid deposits in zooxanthellae function as eye-spots? PLoS One 4, 1-9 (2009).

14. Brychkova, G., Fluhr, R. \& Sagi, M. Formation of xanthine and the use of purine metabolites as a nitrogen source in Arabidopsis plants. Plant Signal. Behav. 3, 999-1001 (2008).

15. Yu, L. et al. Glyoxylate rather than ascorbate is an efficient precursor for oxalate biosynthesis in rice. J. Exp. Bot. 61, 1625-1634 (2010).

16. Gadd, G. M. et al. Oxalate production by fungi: Significance in geomycology, biodeterioration and bioremediation. Fungal Biol. Rev. 28, 36-55 (2014).

17. Winter, G., Todd, C. D., Trovato, M., Forlani, G. \& Funck, D. Physiological implications of arginine metabolism in plants. Front. Plant Sci. 6, 1-14 (2015).

18. Wright, P. A. Nitrogen excretion: Three end products, many physiological roles. J. Exp. Biol. 198, 273-281 (1995).

19. Kourkoulou, A., Pittis, A. A. \& Diallinas, G. Evolution of substrate specificity in the nucleobase-ascorbate transporter (NAT) protein family. Microb. Cell 5, 280-292 (2018).

20. Boswell-Casteel, R. C. \& Hays, F. A. Equilibrative nucleoside transporters - a review. Nuclesides Nucleotides Nucleic Acids 36, 7-30 (2017).

21. Sawada, K. et al. Identification of a vesicular nucleotide transporter. Proc. Natl. Acad. Sci. U. S. A. 105, 5683-5686 (2008). 
22. Bove, M., Cicero, A. F. G., Veronesi, M. \& Borghi, C. An evidence-based review on urate-lowering treatments: implications for optimal treatment of chronic hyperuricemia. Vasc. Health Risk Manag. 13, 23-28 (2017).

23. Ajjawi, I. et al. Lipid production in Nannochloropsis gaditana is doubled by decreasing expression of a single transcriptional regulator. Nat. Biotechnol. 35, 647-652 (2017).

24. Barcytè, D., Pilátová, J., Mojzeš, P. \& Nedbalová, L. The arctic Cylindrocystis (Zygnematophyceae, Streptophyta) green algae are genetically and morphologically diverse and exhibit effective accumulation of polyphosphate. J. Phycol. 56, 217-232 (2020).

25. Moudř́íková, Š. et al. Comparing biochemical and Raman microscopy analyses of starch, lipids, polyphosphate, and guanine pools during the cell cycle of Desmodesmus quadricauda. Cells 10, 1-21 (2021).

26. Eddy, S. R. A probabilistic model of local sequence alignment that simplifies statistical significance estimation. PLoS Comput. Biol. 4, 1-14 (2008).

27. Richter, D. J., Berney, C., Strassert, J. F. H., Burki, F. \& de Vargas, C. EukProt: A database of genome-scale predicted proteins across the diversity of eukaryotic life. bioRxiv 1-11 (2020).

28. Hoang, D. T., Chernomor, O., Von Haeseler, A., Minh, B. Q. \& Vinh, L. S. UFBoot2: Improving the ultrafast bootstrap approximation. Mol. Biol. Evol. 35, 518-522 (2018).

29. Katoh, K. \& Standley, D. M. MAFFT multiple sequence alignment software version 7: Improvements in performance and usability. Mol. Biol. Evol. 30, 772-780 (2013).

30. Hall, T. BioEdit: a user-friendly biological sequence alignment editor and analysis program for Windows 95/98/NT. Nucleic Acids Symp. Ser. 41, 95-98 (1999).

31. Stamatakis, A. RAxML version 8: A tool for phylogenetic analysis and post-analysis of large phylogenies. Bioinformatics 30, 1312-1313 (2014).

32. Keane, T. M., Creevey, C. J., Pentony, M. M., Naughton, T. J. \& McInerney, J. O. Assessment of methods for amino acid matrix selection and their use on empirical data shows that ad hoc assumptions for choice of matrix are not justified. BMC Evol. Biol. 6, 1-17 (2006).

33. Young, J. D., Yao, S. Y. M., Baldwin, J. M., Cass, C. E. \& Baldwin, S. A. The human concentrative and equilibrative nucleoside transporter families, SLC28 and SLC29. Mol. Aspects Med. 34, 529-547 (2013).

34. Adl, S. M. et al. Revisions to the classification, nomenclature, and diversity of eukaryotes. J. Eukaryot. Microbiol. 66, 4-119 (2019).

35. Archibald, J. M. et al. Handbook of the Protists. Handbook of the Protists (Springer International Publishing, Switzerland, 2017).

36. Oborník, M. Photoparasitism as an intermediate state in the evolution of apicomplexan parasites. Trends Parasitol. 36, 727-734 (2020).

37. Hehenberger, E., Burki, F., Kolisko, M. \& Keeling, P. J. Functional relationship between a dinoflagellate host and its diatom endosymbiont. Mol. Biol. Evol. 33, 2376-2390 (2016).

38. Litaker, R. W. et al. Seasonal niche strategy of the bloom-forming dinoflagellate Heterocapsa triquetra. Mar. Ecol. Prog. Ser. 232, 45-62 (2002).

39. Li, Z., Ma, X., Li, A. \& Zhang, C. A novel potential source of $\beta$-carotene: Eustigmatos cf. polyphem (Eustigmatophyceae) and pilot $\beta$-carotene production in bubble column and flat panel photobioreactors. Bioresour. Technol. 117, 257-63 (2012).

40. Zienkiewicz, A. et al. The microalga Nannochloropsis during transition from quiescence 
to autotrophy in response to nitrogen availability. Plant Physiol. 182, 819-839 (2020).

41. Priyadarshani, I. \& Rath, B. Commercial and industrial applications of micro algae - A review. J. Algal Biomass Util. 3, 89-100 (2012).

42. Smith, S. R. et al. Evolution and regulation of nitrogen flux through compartmentalized metabolic networks in a marine diatom. Nat. Commun. 10, 1-14 (2019).

43. Hedley, R. H. \& Bertaud, W. S. Electron-microscopic observations of Gromia oviformis (Sarcodina). J. Protozool. 9, 79-87 (1962).

44. Paasche, E. A review of the coccolithophorid Emiliania huxleyi (Prymnesiophyceae), with particular reference to growth, coccolith formation, and calcification-photosynthesis interactions. Phycologia 40, 503-529 (2001).

45. Hoef-Emden, K. \& Melkonian, M. Revision of the genus Cryptomonas (Cryptophyceae): A combination of molecular phylogeny and morphology provides insights into a longhidden dimorphism. Protist 154, 371-409 (2003).

46. Kumar, D., Kvíderová, J., Kaštánek, P. \& Lukavský, J. The green alga Dictyosphaerium chlorelloides biomass and polysaccharides production determined using cultivation in crossed gradients of temperature and light. Eng. Life Sci. 17, 1030-1038 (2017).

47. Barcyte, D., Hodac, L., Nedbalova, L. \& Elster, J. Chloromonas arctica sp. Nov., a psychrotolerant alga from snow in the high arctic (Chlamydomonadales, Chlorophyta). Int. J. Syst. Evol. Microbiol. 68, 851-859 (2018).

48. Lelandais, G. et al. Ostreococcus tauri is a new model green alga for studying iron metabolism in eukaryotic phytoplankton. BMC Genomics 17, 1-23 (2016).

49. De Vries, J., Curtis, B. A., Gould, S. B. \& Archibald, J. M. Embryophyte stress signaling evolved in the algal progenitors of land plants. Proc. Natl. Acad. Sci. U. S. A. 115, E3471E3480 (2018).

50. Nakagaki, T., Kobayashi, R., Nishiura, Y.\& Ueda, T. Obtaining multiple separate food sources: Behavioural intelligence in the Physarum plasmodium. Proc. R. Soc. B Biol. Sci. 271, 2305-2310 (2004).

51. Marciano-Cabral, F. \& Cabral, G. Acanthamoeba spp. as agents of disease in humans. Clin. Microbiol. Rev. 16, 273-307 (2003).

52. Gur, D., Palmer, B. A., Weiner, S. \& Addadi, L. Light manipulation by guanine crystals in organisms: biogenic scatterers, mirrors, multilayer reflectors and photonic crystals. $A d v$. Funct. Mater. 27, 1-13 (2017).

53. Roush, A. H., Questiaux, L. M. \& Domnas, A. J. The active transport and metabolism of purines in the yeast, Candida utilis. J. Cell. Comp. Physiol. 54, 275-286 (1959).

54. Flegontova, O. et al. Environmental determinants of the distribution of planktonic diplonemids and kinetoplastids in the oceans. Environ. Microbiol. 22, 4014-4031 (2020).

55. Flegontova, O. et al. Extreme diversity of diplonemid eukaryotes in the Ocean. Curr. Biol. 26, 3060-3065 (2016).

56. Tsaousis, A. D., Nývltová, E., Šuták, R., Hrdý, I. \& Tachezy, J. A Nonmitochondrial hydrogen production in Naegleria gruberi. Genome Biol. Evol. 6, 792-799 (2014).

57. Maaß, A. \& Radek, R. The gut flagellate community of the termite Neotermes cubanus with special reference to Staurojoenina and Trichocovina hrdyi nov. gen. nov. sp. Eur. J. Protistol. 42, 125-141 (2006).

58. Lax, G. et al. Hemimastigophora is a novel supra-kingdom-level lineage of eukaryotes. Nature 564, 410-414 (2018).

59. Brown, M. W. et al. Phylogenomics places orphan protistan lineages in a novel eukaryotic 
super-group. Genome Biol. Evol. 10, 427-433 (2018).

60. Ehrlich, H. et al. Multiphase biomineralization: enigmatic invasive siliceous diatoms produce crystalline calcite. Adv. Funct. Mater. 26, 2503-2510 (2016).

61. Romann, J. et al. Wavelength and orientation dependent capture of light by diatom frustule nanostructures. Sci. Rep. 5, 1-6 (2015).

62. Lipej, L., Orlando-Bonaca, M. \& Mavrič, B. Biogenic formations in the Slovenian sea. (2016).

63. Weiner, S. \& Addadi, L. Crystallization pathways in biomineralization. Annu. Rev. Mater. Res. 41, 21-40 (2011).

64. Lenzenweger, R. Algen mit Kristallschmuck. Mikrokosmos 91, 280 (2002).

65. Martignier, A. et al. Marine and freshwater micropearls: Biomineralization producing strontium-rich amorphous calcium carbonate inclusions is widespread in the genus Tetraselmis (Chlorophyta). Biogeosciences 15, 6591-6605 (2018).

66. Odum, H. T. Notes on the strontium content of sea water, celestite Radiolaria, and strontianite snail shells. Science (80-. ). 114, 211-213 (1951).

67. Bütschli, O. Über die chemische Natur der Skelettsubstanz der Acantharia. Zool. Anz. 30, 784-789 (1906).

68. Brook, A. J., Fotheringham, A., Bradly, J. \& Jenkins, A. Barium accumulation by desmids of the genus Closterium (Zygnemaphyceae). Br. Phycol. J. 15, 261-264 (1980).

69. Kreger, D. R. \& Boeré, H. Some observations on barium sulphate in Spirogyra. Acta Bot. Neerl. 18, 143-151 (1969).

70. Nakata, P. A. Plant calcium oxalate crystal formation, function, and its impact on human health. Front. Biol. (Beijing). 7, 254-266 (2012).

71. Maass, D., Arango, J., Wüst, F., Beyer, P. \& Welsch, R. Carotenoid crystal formation in Arabidopsis and carrot roots caused by increased phytoene synthase protein levels. PLoS One 4, 1-12 (2009).

72. Aboal, M. \& Werner, O. Morphology, fine structure, life cycle and phylogenetic analysis of phyllosiphon arisari, a siphonous parasitic green alga. Eur. J. Phycol. 46, 181-192 (2011).

73. Škaloud, P., Friedl, T., Hallmann, C., Beck, A. \& Dal Grande, F. Taxonomic revision and species delimitation of coccoid green algae currently assigned to the genus Dictyochloropsis (Trebouxiophyceae, Chlorophyta). J. Phycol. 52, 599-617 (2016).

74. Xiao, H. et al. The study on starch granules by using darkfield and polarized light microscopy. J. Food Compos. Anal. 92, 1-7 (2020).

75. Kreger, D. R. \& van der Veer, J. Paramylon in a Chrysophyte. Acta Bot. Neerl. 19, 401402 (1970).

76. Li, J. et al. Probing the cyanobacterial microcystis gas vesicles after static pressure treatment: A potential in situ rapid method. Sensors 20, 1-17 (2020).

77. Abraham, Y. \& Elbaum, R. Quantification of microfibril angle in secondary cell walls at subcellular resolution by means of polarized light microscopy. New Phytol. 197, 10121019 (2013).

78. Patching, S. G. Recent developments in nucleobase cation symporter-1 (NCS1) family transport proteins from bacteria, archaea, fungi and plants. J. Biosci. 43, 797-815 (2018).

79. Liu, X., Qian, W., Liu, X., Qin, H. \& Wang, D. Molecular and functional analysis of hypoxanthine-guanine phosphoribosyltransferase from Arabidopsis thaliana. New Phytol. 175, 448-461 (2007). 
80. Prokopchuk, G. et al. Morphological, ultrastructural, motility and evolutionary characterization of two new Hemistasiidae species. Protist 170, 259-282 (2019).

81. Bischoff, H. W. \& Bold, H. C. Phycological Studies IV. Some Soil Algae from Enchanted Rock and Related Algal Species. (University of Texas Publication No. 6318, 1963).

82. Votýpka, J. et al. Kentomonas gen. n., a new genus of endosymbiont-containing trypanosomatids of Strigomonadinae subfam. n. Protist 165, 825-838 (2014).

83. Andersen, R. A., Morton, S. L. \& Sexton, J. P. Provasoli Guillard National Center for Culture of Marine Phytoplankton 1997 - list of strains. J. Phycol. 33, 1-75 (1997).

84. Guillard, R. R. L. Culture of phytoplankton for feeding marine invertebrates. in Culture of marine invertebrate animals 29-60 (Plenum Press, 1975).

85. Park, J. S. Effects of different ion compositions on growth of obligately halophilic protozoan Halocafeteria seosinensis. Extremophiles 16, 161-164 (2012).

86. Bertani, G. Studies on lysogenesis. I. The mode of phage liberation by lysogenic Escherichia coli. J. Bacteriol. 62, 293-300 (1951).

87. Fulton, C. Axenic cultivation of Naegleria gruberi. Requirement for methionine. Exp. Cell Res. 88, 365-370 (1974).

88. Samson, R. A., Hoekstra, E. S. \& Oorschot, C. A. N. Van. Introduction to food-borne fungi. (Centraalbureau voor Schimmelcultures, 1981).

89. Murashige, T. \& Skoog, F. A revised medium for rapid growth and bio assays with tobacco tissue cultures. Physiol. Plant. 15, 474-497 (1962).

90. Neff, R. J. \& Neff, R. H. Induction of synchronous division in amoebae. in Synchrony in Cell Division and Growth 213-246 (Wiley Interscience, 1964).

91. Burgess, L. W., Liddell, C. M. \& Summerell, B. A. Laboratory manual for fusarium research: incorporating a key and descriptions of common species found in Australasia. (The University of Sydney, 1988).

92. Chávez, L. A., Balamuth, W. \& Gong, T. A light and electron microscopical study of a new, polymorphic free-living amoeba, Phreatamoeba balamuthi n. g., n. sp. J. Protozool. 33, 397-404 (1986).

93. Diamond, L. S. Establishment of various trichomonads of animals and man in axenic cultures. J. Parasitol. 43, 488-490 (1957). 


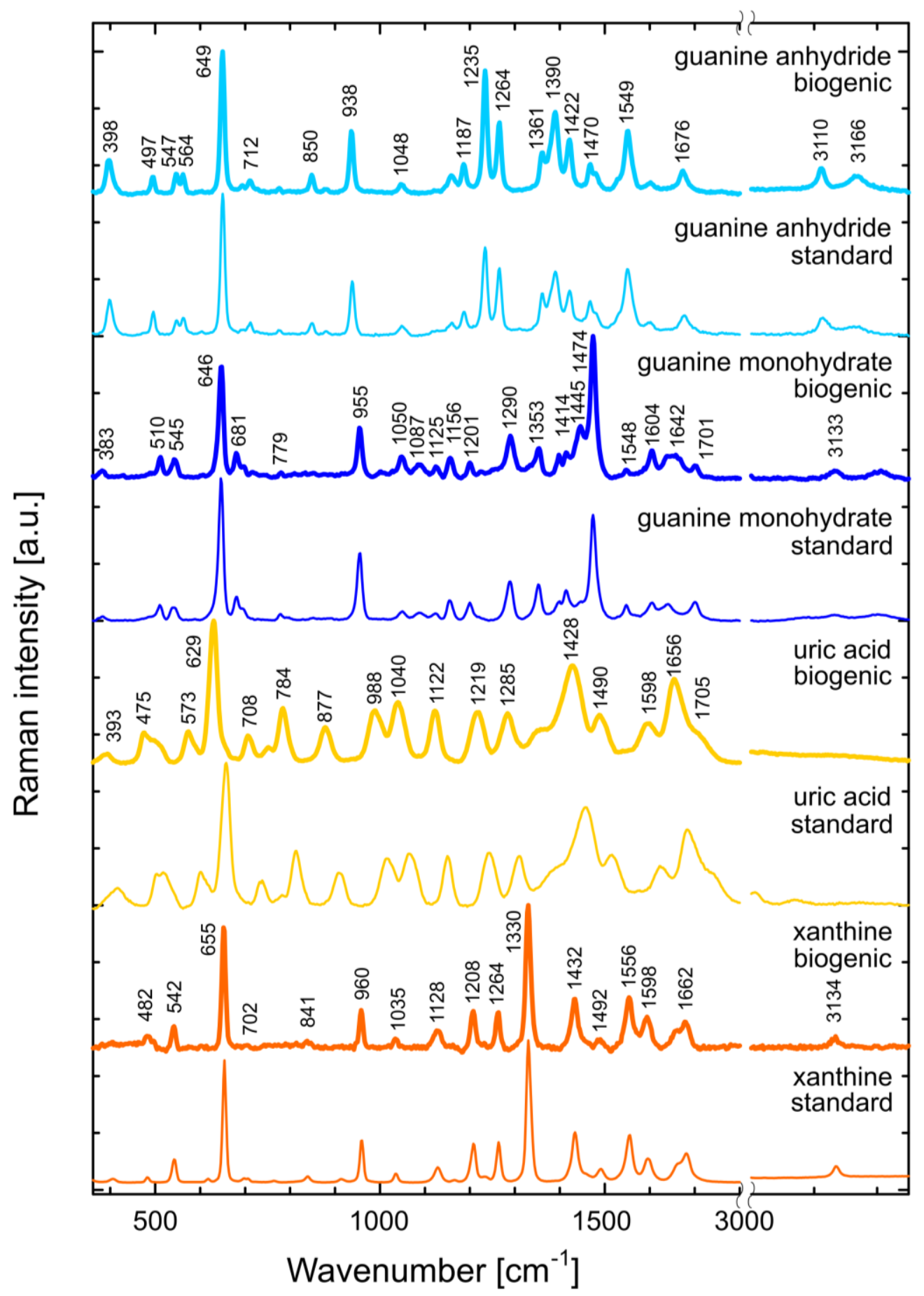

\section{Fig. S1.}

Representative Raman spectra of biogenic purine crystalline inclusions measured in biological species followed by their respective standards of pure chemical compounds. Biogenic crystals have been spectrally extracted directly from measured species. Standards of guanine anhydride and xanthine have been measured as suspension of pure compounds in water, guanine monohydrate and uric acid has been recrystallized from $4 \%$ dimethylamine water solution. 


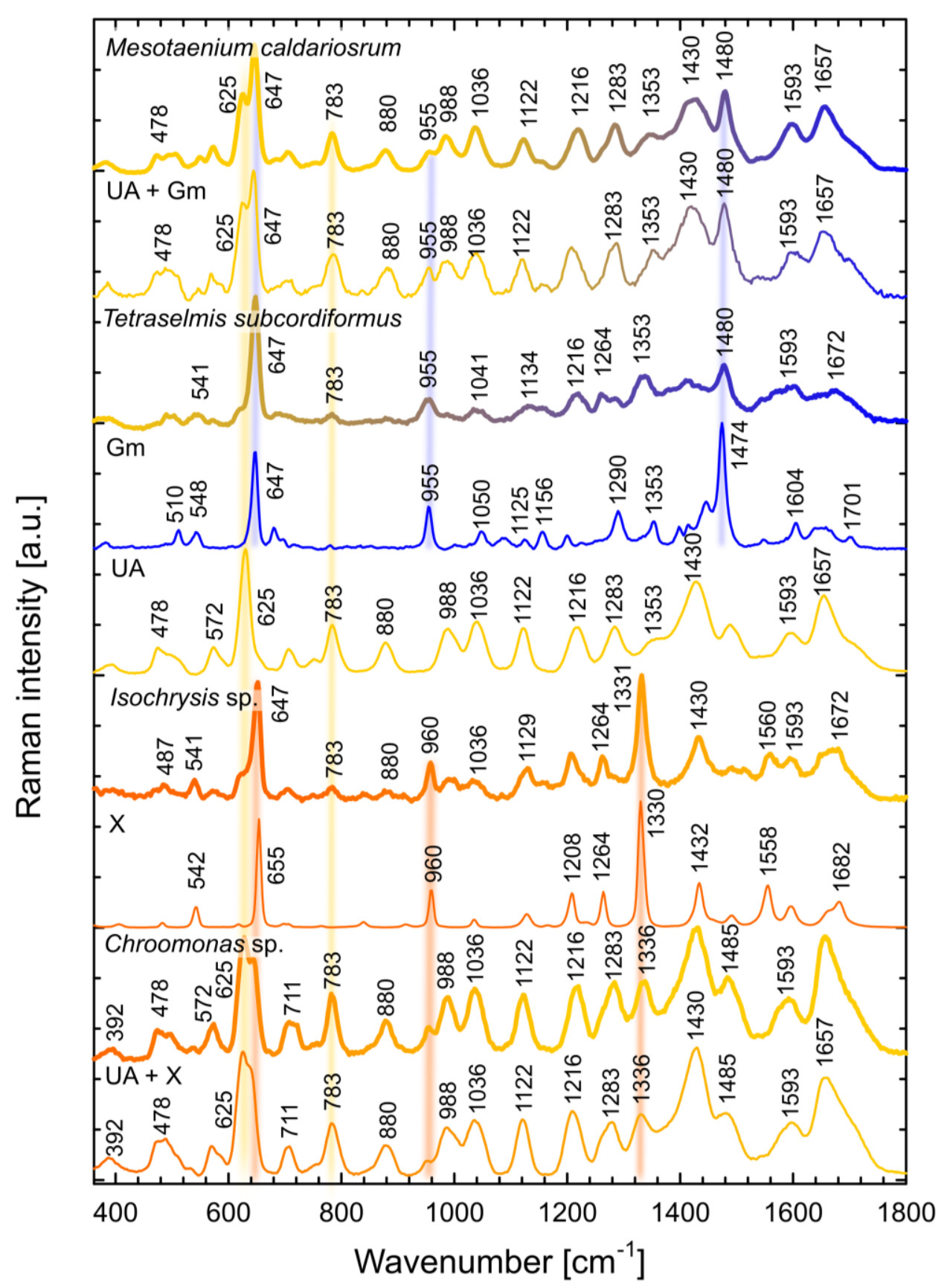

Fig. S2.

Representative Raman spectra of biogenic purine inclusions forming mixtures of uric acid (UA) and guanine monohydrate $(\mathrm{Gm})$ in different proportions with a dominance of the former in the case of Mesotaenium caldariorum, or the latter in Tetraselmis subcordiformis. Xanthine (X) dominates the crystals with uric acid admixtures found in Isochrysis sp., whereas Chroomonas sp. has higher proportions of uric acid over xanthine. The reference spectra of pure substances are shown along with those of mixtures of "UA $+\mathrm{Gm}$ " and "UA $+\mathrm{X}$ " recrystallized from 4\% dimethylamine solution, as Raman spectra of purine mixtures exhibit some spectral shifts and changes in relative intensities compared to the pure substances. 


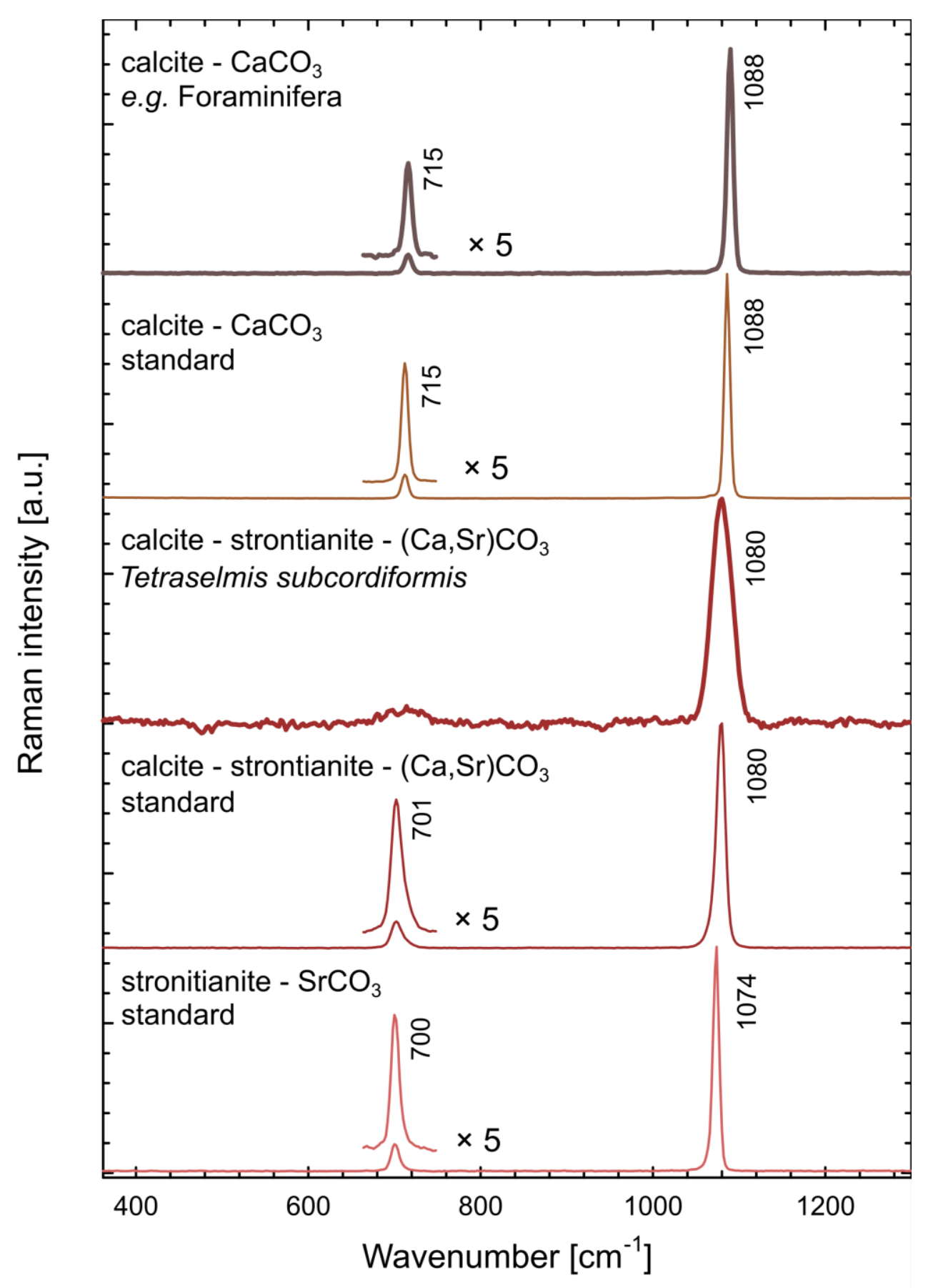

Fig. S3.

Representative Raman spectra of carbonate minerals observed in inspected species, all of them strongly polarize light: calcite or calcium carbonate $\left(\mathrm{CaCO}_{3}\right)$ found in various diatoms, Foraminifera, multicellular Rhodophytes and seaweed with respective standard and calcite with admixtures of strontianite $\left(\mathrm{SrCO}_{3}\right)$ or strontium carbonate $(\mathrm{Ca}, \mathrm{Sr}) \mathrm{CO}_{3}$ found in Tetraselmis sp. with respective standards of pure chemical substances. 


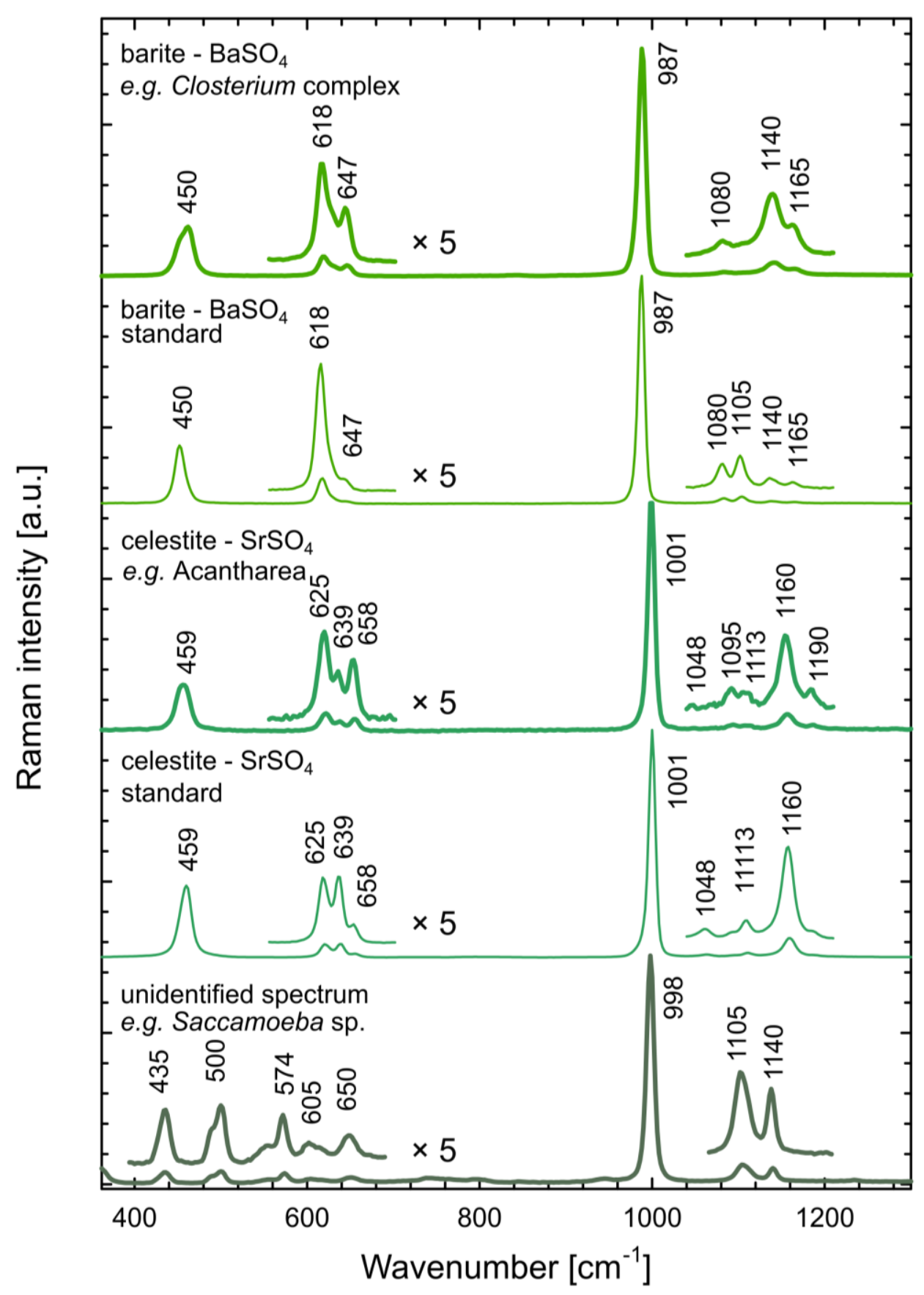

\section{Fig. S4.}

Representative Raman spectra of sulfate minerals observed in inspected species followed by respective standards of pure chemical substances, all of them faintly polarize light: baryte $\left(\mathrm{BaSO}_{4}\right)$ found in Closterium peracerosum-strigosum-littorale complex, Cosmarium sp., Spirogyra sp., celestite $\left(\mathrm{SrSO}_{4}\right)$ found in skeletons of Acantharea, and unidentified spectra of sulfate resembling minerals mixed with lipophilic organic matter found in Saccamoeba sp. Raman spectra of sulfates show variability in dependence of crystal orientation and minor admixtures of other salts. 


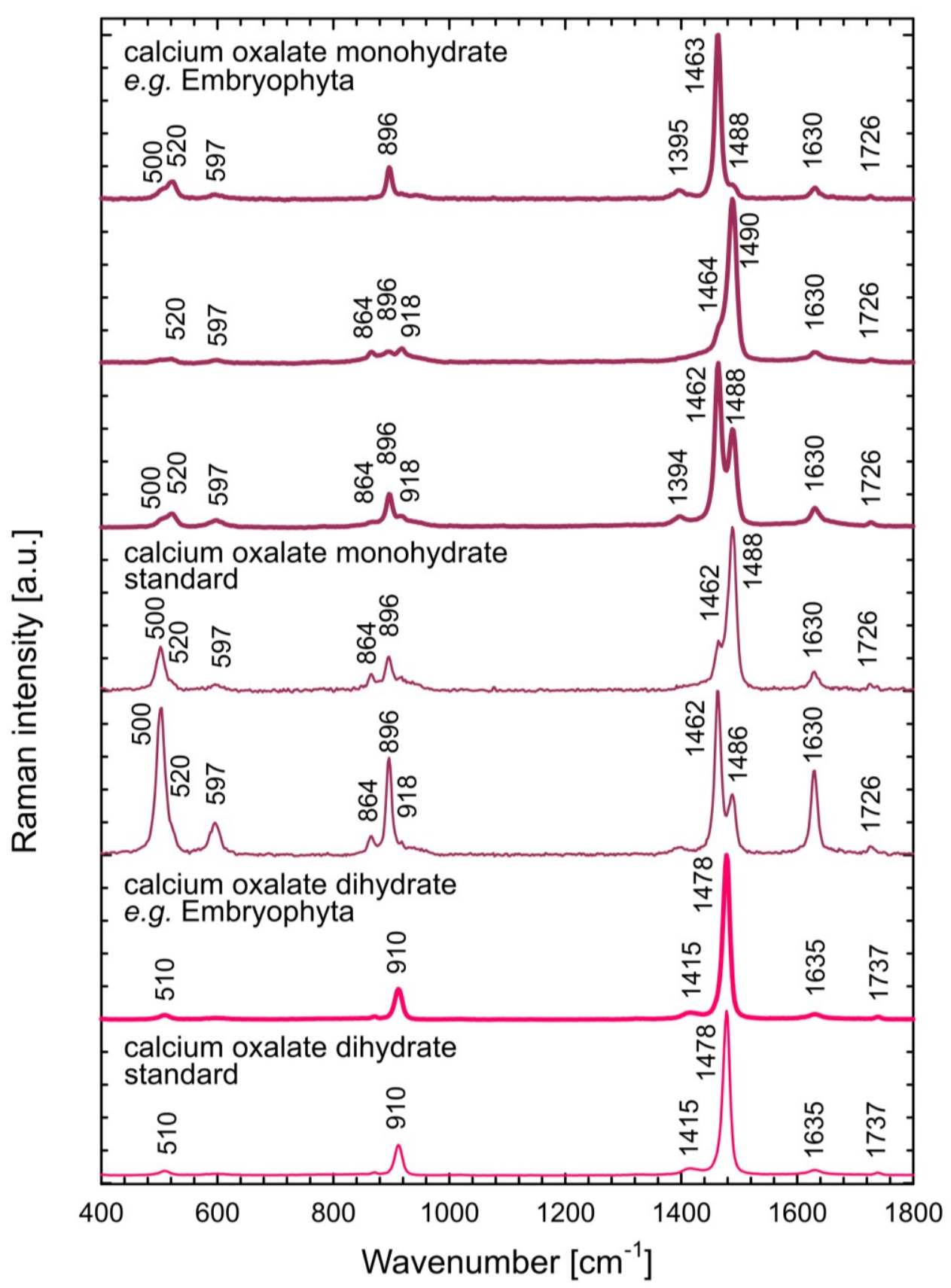

\section{Fig. S5.}

Representative Raman spectra of organic crystals polarizing light observed in inspected species followed by respective standards of pure chemical substances. The crystals of calcium oxalate monohydrate strongly polarize Raman signal - the major peaks interchange their relative intensities according to the crystal orientation with respect of polarization plane of the excitation beam. It was found in Coleochaetophyceae, Zygnematophyceae, Embryophyta. The calcium oxalate dihydrate was found in Embryophyta. 


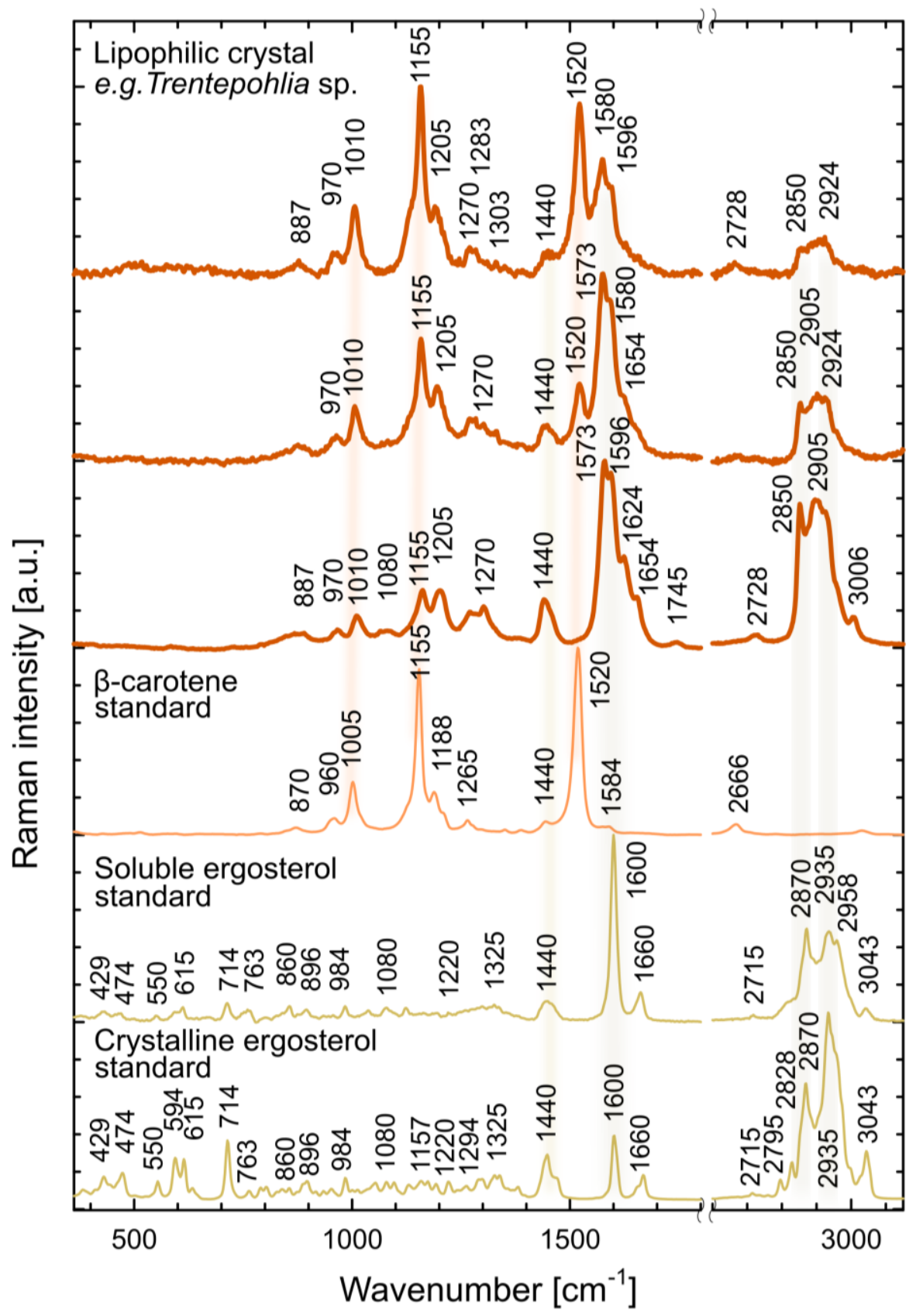

\section{Fig. S6.}

Representative Raman spectra of organic crystals polarizing light observed in inspected species followed by respective standards of pure chemical substances: lipophilic crystalline mixtures of carotenoids and sterols found in ulvophytes Trentepohlia sp. and Scotinosphaera giberosa, rhodophyte Asterocytis ramonsa and cyanobacteria Oscillatoria sp. Raman measurements using a high-intensity laser beam lead to photo-degradation of carotenoids present in the structure, thus their Raman signal decreases over time and allows observation of other admixtures in greater detail. We failed to find a precisely matching standard for sterols forming these lipophilic crystals; the biogenic crystals may contain a complex mixture of various chemical species. 


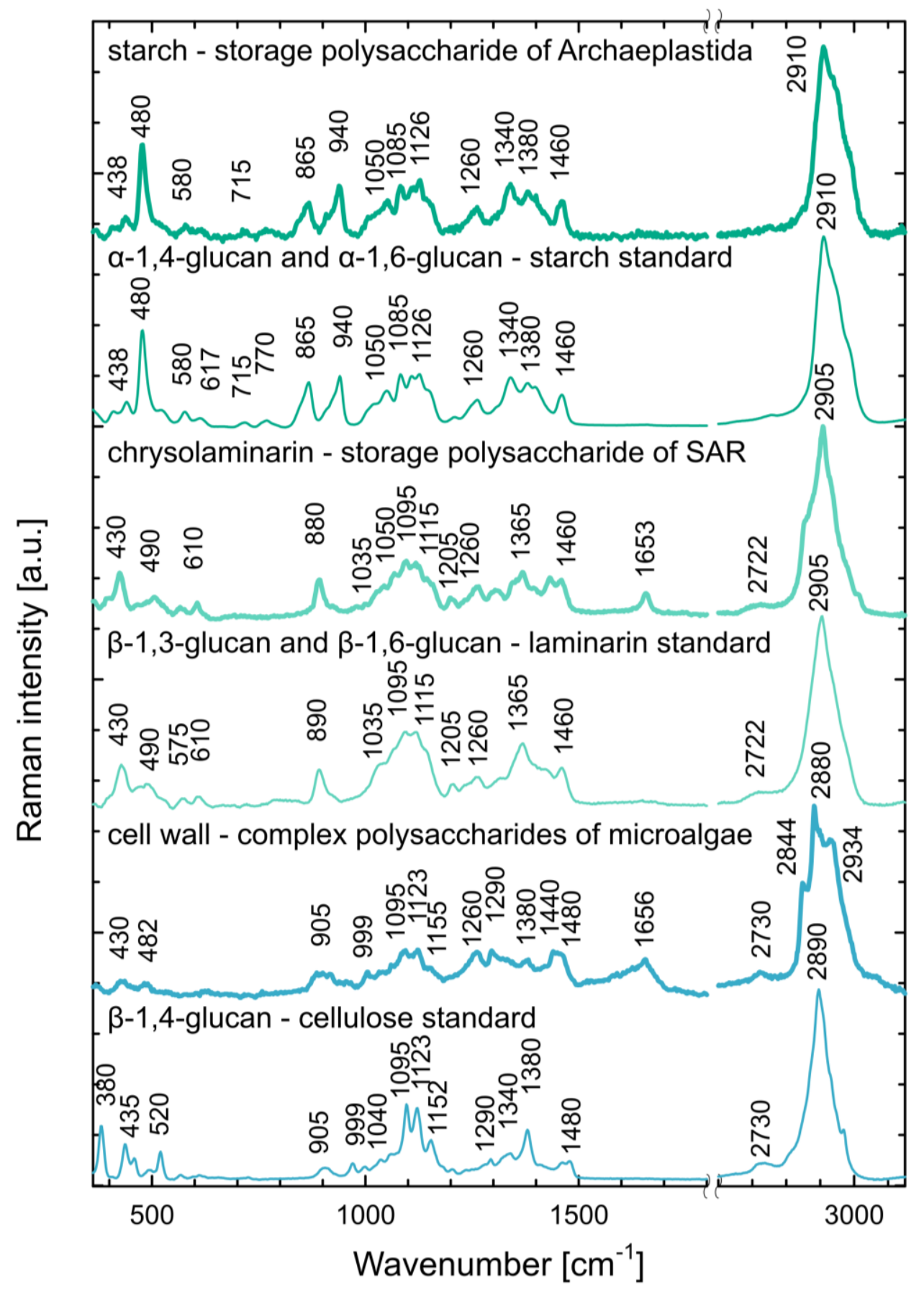

Fig. S7.

Representative Raman spectra of light polarizing polysaccharides in inspected species with respective standards of pure chemical substances: starch - storage polysaccharide of $\alpha$-1,4-glucan and $\alpha$-1,6-glucan found in Archaeplastida, chrysolaminarin - storage polysaccharide of $\beta$-1,3glucan and $\beta$-1,6-glucan found in SAR, cellulose - structure polysaccharide of $\beta$-1,4-glucan forming cell walls of various microalgae (both Archaeplastida and SAR). 


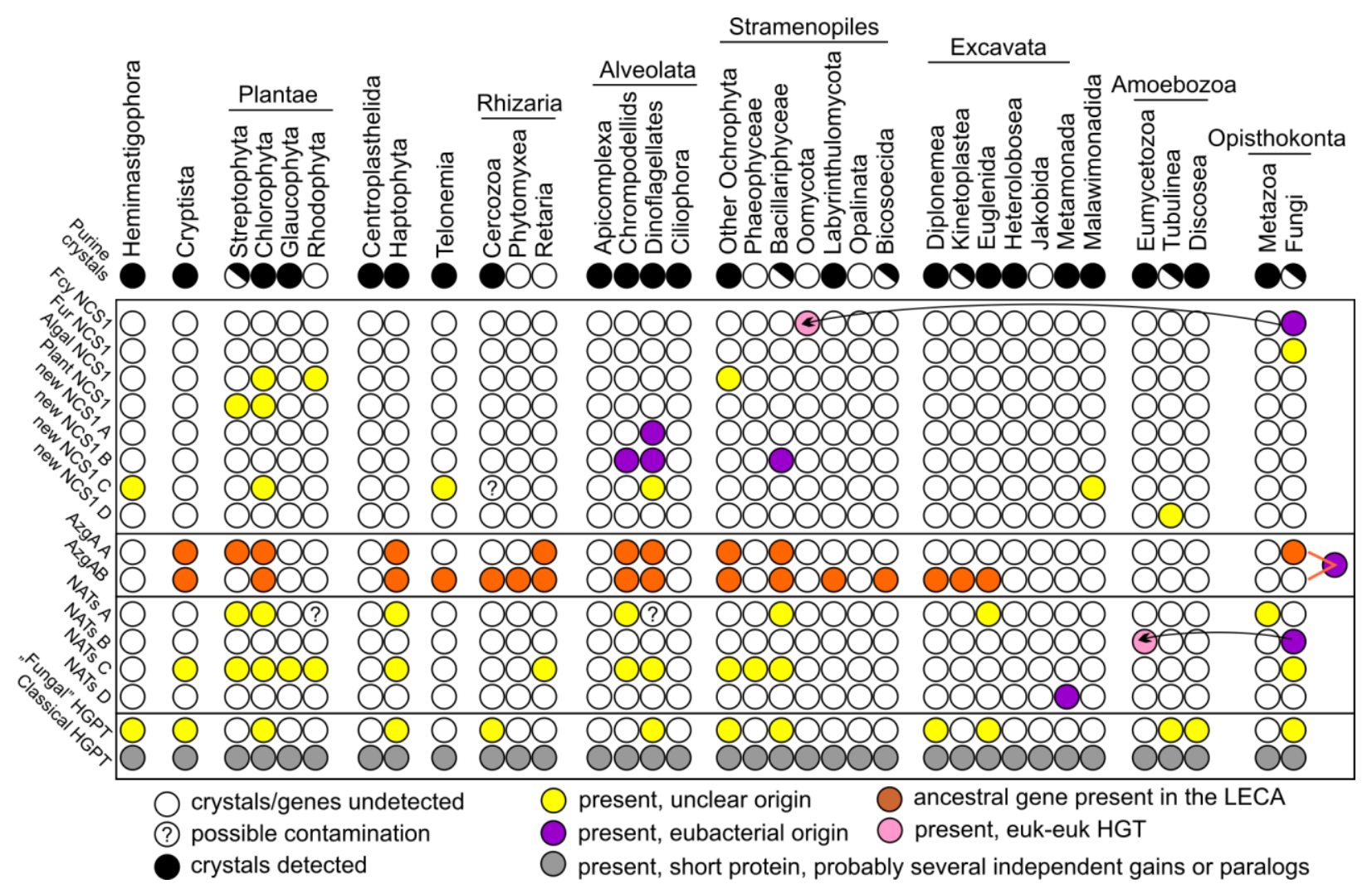

Fig. S8.

Summary table of phylogenetic distribution of the purine transporters: NCS1, NAT, AzgA, and the metabolic enzyme of salvage pathway - HGPT. There are notions on horizontal gene transfer (HGT) in two cases. In the case of AzgA we anticipate a possible origin in last eukaryotic common ancestor (LECA). 


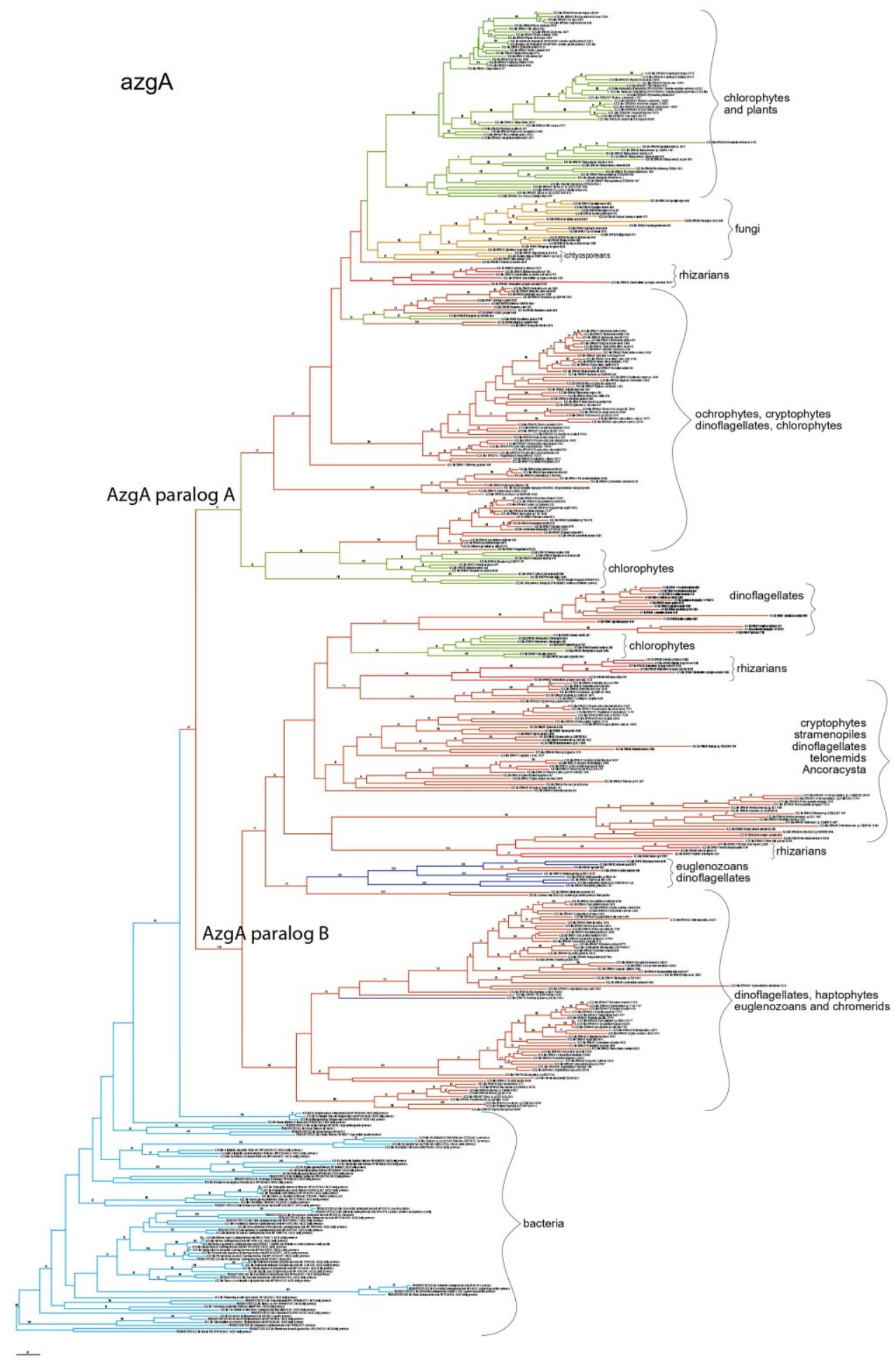

\section{Fig. S9.}

Maximum likelihood tree of AzgA as inferred from amino acid sequences (395 aa). Numbers above branches indicate ML bootstrap support (200 replicates). 


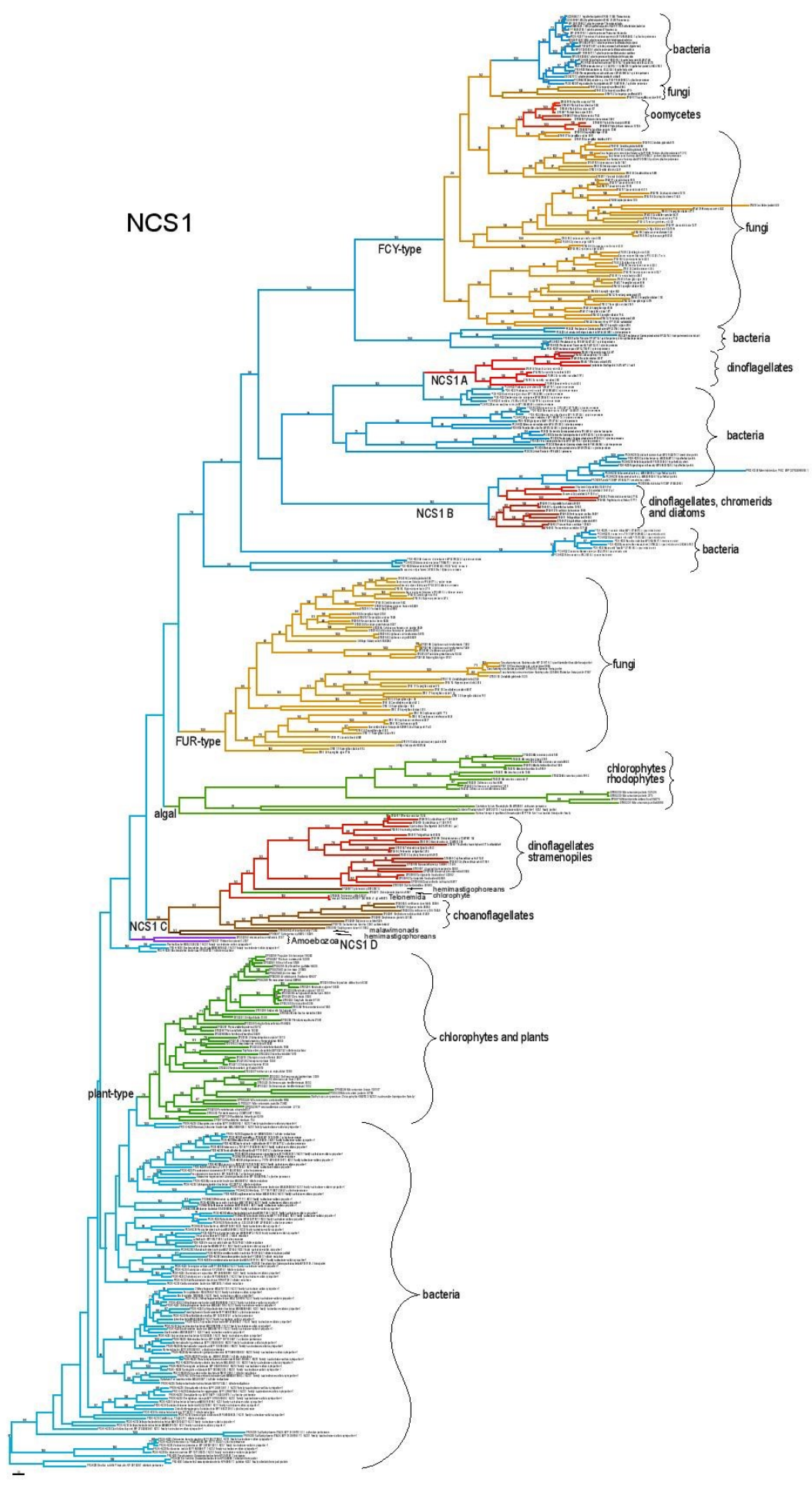

Fig. S10.

Maximum likelihood tree of nucleobase-cation symporter 1 (NCS1) as inferred from amino acid sequences (463 aa positions). Numbers above branches indicate bootstrap support (200 replicates). 


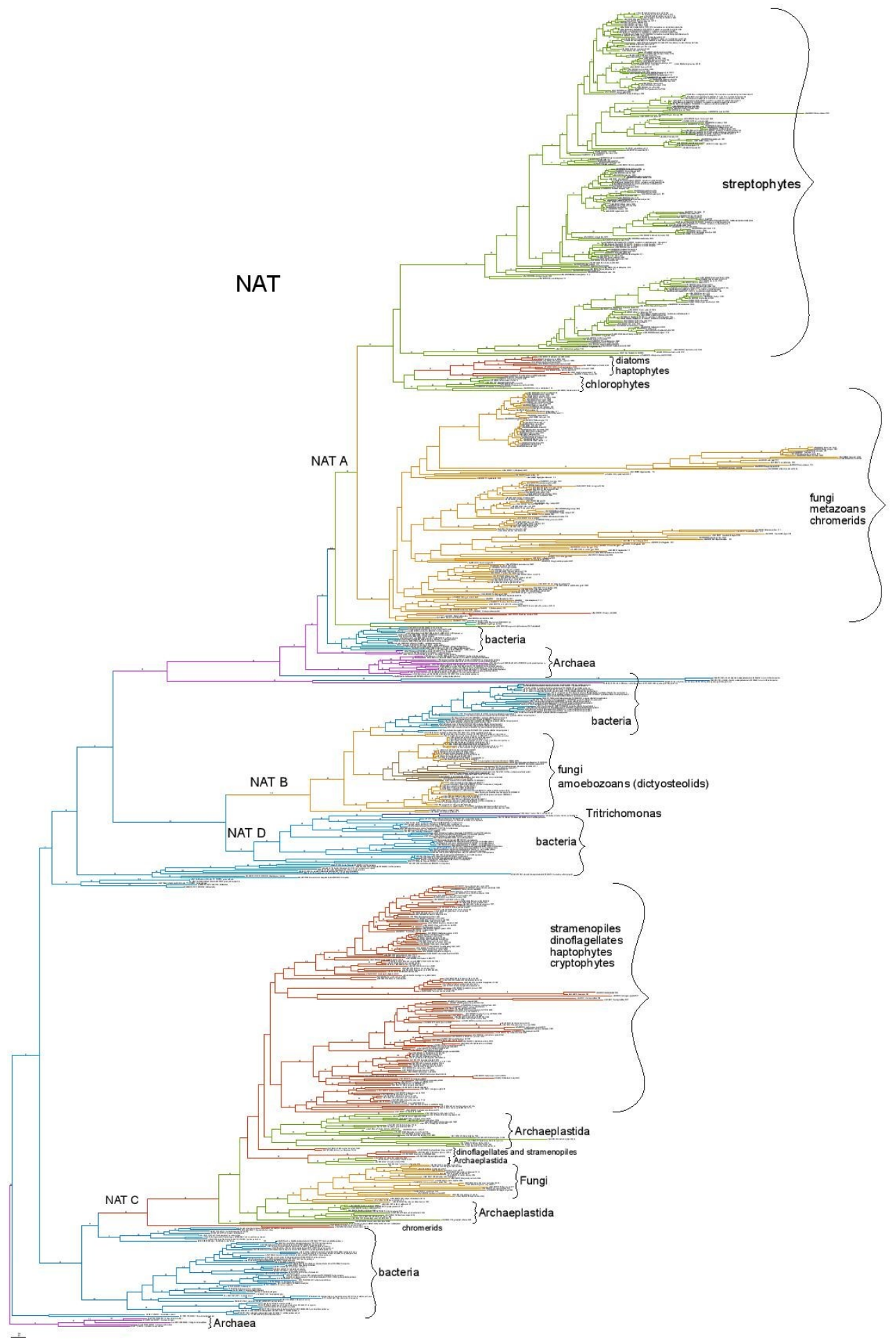

\section{Fig. S11.}

Maximum likelihood tree of nucleobase-ascorbate transporter (NAT) as inferred from amino acid sequences (385 aa). Numbers above branches indicate bootstrap support (200 replicates). 


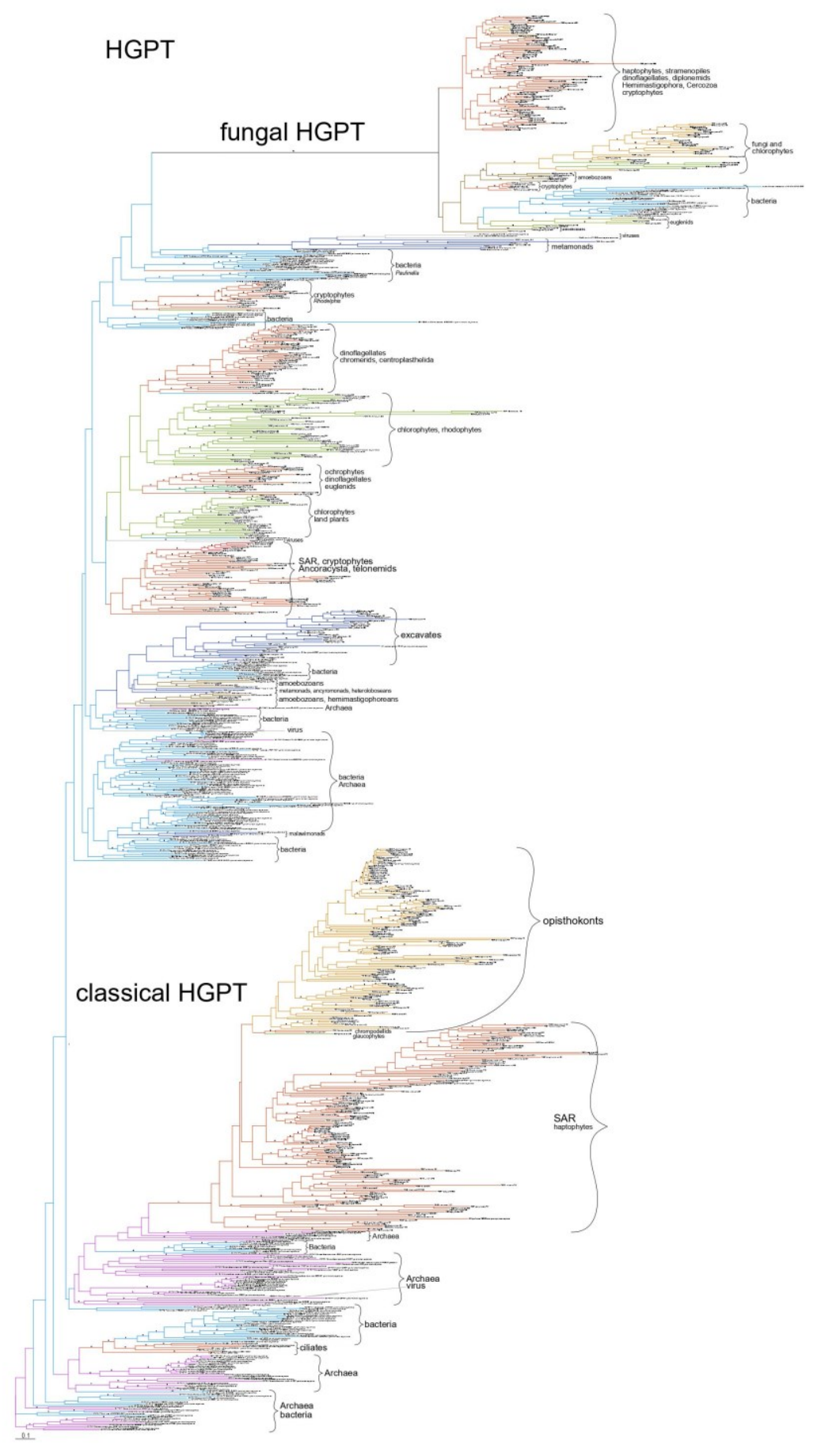

\section{Fig. S12.}

Maximum likelihood tree of hypoxanthine-guanine phosphoribosyl transferase (HGPT) as inferred from amino acid sequences (171 aa). Numbers above branches indicate bootstrap support (200 replicates). 
Table S1. Overview of the cell inclusions present in eukaryotes and cyanobacteria and their identification via Raman microscopy and light polarization. Species are listed according to their taxonomic classification and then alphabetically - species positively tested for purine inclusions (in black) and not positively tested for purine inclusions (in grey); capital letters in brackets refer to the phylogeny scheme in Fig. 1; (b) stands for biotechnologically important species and $(\mathrm{m})$ for model organism. Habitat and -trophy: E - Endobiont, F - freshwater, Hp halophilic, $\mathrm{M}$ - marine, $\mathrm{S}$ - snow, $\mathrm{T}$ - terrestrial; a - autotroph, $\mathrm{h}$ - heterotroph, $\mathrm{p}$ - parasite. Culture collections: ATCC - American Type Culture Collection, Manassas, USA; CAUP Culture Collection of Algae of Charles University in Prague, Czech Republic; CCALA - Culture collection of Autotrophic Organisms of the Institute of Botany of the AS CR, Třeboň, Czech Republic; CCAP - Culture Collection of Algae and Protozoa, Orban, UK; CCF - Culture collection of Fungi, Praha, Czech Republic; CCMP - Culture Collection of Marine Phytoplankton - part of NCMA: NCMA - National Center for Marine Algae and Microbiota, Bigelow, USA; CRC - Chlamydomonas Resource Center, University of Minnesota, St. Paul, USA; CVCC - China Veterinary Culture Collection Center, National Control Institute of Veterinary Bio-products and Pharmaceutical (NCIVBP), Ministry of Agriculture, Beijing, China; DBM - Department of Biochemistry and Microbiology at University of Chemistry and Technology, Prague, Czech Republic; EUROSCARF - EUROpean Saccharomyces Cerevisiae Archive for Functional analysis, Scientific Research and Development GmbH, Oberursel, Germany; NIES - National Institute for Environmental Studies, Tsukuba, Japan; NCMA - CCMP - National Center for Marine Algae and Microbiota, Culture Collection of Marine Phytoplankton, Bigelow, USA; priv - private collection; RCC - Roscoff Culture Collection, Roscoff, France; SAG - Culture Collection of Algae at Goettingen University, Germany; UTEX - University of Texas at Austin, USA

\section{Cultivation method:}

- - no cultivation prior to observations and measurements (in case of environmental samples) ASW-D - Artificial Sea Water for Diplonemids according to ${ }^{80}, 20^{\circ} \mathrm{C}$

ASW-L - Artificial Sea Water for Labyrinthulomycetes: 3,5\% artificial sea water supplied with $1 \mathrm{~g}$ of yeast extract and $10 \mathrm{~g}$ of glucose, $25^{\circ} \mathrm{C}$

BBM - Basal Bold's Medium according to ${ }^{81}, 20-25^{\circ} \mathrm{C}, 16: 8 \mathrm{~h}$ light:dark cycle

BHI - Brain Heart Infusion medium as described in ${ }^{82}$

DY-IV - medium according to ${ }^{83}, 15^{\circ} \mathrm{C}, 12: 12 \mathrm{~h}$ light:dark cycle

$\mathrm{f} / 2$ - medium according to ${ }^{84}, 20-25^{\circ} \mathrm{C}, 16: 8 \mathrm{~h}$ light:dark cycle

$\mathrm{f} / 2 \mathrm{~B}-\mathrm{f} / 2$ medium according to ${ }^{84}$ with addition of sterile barley grain, $20-25^{\circ} \mathrm{C}$

Fw - ATCC 802 Sonneborn's Paramecium medium, 20-25 ${ }^{\circ} \mathrm{C}$

FwAM - Fresh Water Amoeba Medium - ATCC medium 997, 20-25 ${ }^{\circ} \mathrm{C}$

FwB1 - 25\% ATCC 802 Sonneborn's Paramecium medium, with addition of sterile barley grain, $20-25^{\circ} \mathrm{C}$

FwB2 - 25\% ATCC 802 Sonneborn's Paramecium medium, with addition of sterile barley grain, $17^{\circ} \mathrm{C}$

HM-V - Halophiles Medium number V according to ${ }^{85}$ with addition of sterile barley grain, 20$25^{\circ} \mathrm{C}$

$\mathrm{JM}-\mathrm{ATCC} 1525,25^{\circ} \mathrm{C}$

LB - 3\% Lysogeny Broth medium according to ${ }^{86}, 25^{\circ} \mathrm{C}$

LBM $-3 \%$ Lysogeny Broth medium according to ${ }^{86}$ in Artificial Sea Water, $25^{\circ} \mathrm{C}$ 
M7 - medium according to ${ }^{87}, 25^{\circ} \mathrm{C}$

MAM - Marine Amoeba Medium - ATCC medium 994, $25^{\circ} \mathrm{C}$

MEA - Malt Extract Agar according to ${ }^{88}, 25^{\circ} \mathrm{C}$

MS-1 - Murashige and Skoog liquid medium according to ${ }^{89}, 25^{\circ} \mathrm{C}, 16: 8 \mathrm{~h}$ light:dark cycle

MS-2 - Murashige and Skoog solid medium according to ${ }^{89}, 25^{\circ} \mathrm{C}, 16: 8 \mathrm{~h}$ light:dark cycle

Neff - medium of Neff and Neff according to ${ }^{90}, 25^{\circ} \mathrm{C}$

$\mathrm{OG}$ - oat grain on cellulose paper kept in $100 \%$ humidity, $25^{\circ} \mathrm{C}$

PDA - Potato Dextrose Agar according to ${ }^{91}, 25^{\circ} \mathrm{C}$

PYGC - Proteose peptone - Yeast extract - Glucose-Cysteine medium according to ${ }^{92}, 25^{\circ} \mathrm{C}$

TYI - ATCC 2695 Keister's Modified TYI-S-33 medium, $37{ }^{\circ} \mathrm{C}$

TYM - medium modified according to ${ }^{93}$ by an overlay of inactivated horse serum, $25^{\circ} \mathrm{C}$

YPAD - ATCC 1069 medium, $37^{\circ} \mathrm{C}$

$\dagger-$ fixed cells

* - supplementary guanine added (approximately $30 \mu \mathrm{M}$ final concentration), sterilized by

$0.22 \mu \mathrm{m}$ filtration

${ }^{\#}$ - same species has been measured previously for publication in ${ }^{8}$ 


\begin{tabular}{|c|c|c|c|c|c|c|}
\hline $\begin{array}{l}\text { Cult. } \\
\text { coll. }\end{array}$ & Code & Species & $\begin{array}{l}\text { Habitat } \\
\text {-trophy }\end{array}$ & Taxonomic classification & $\begin{array}{l}\text { Raman } \\
\text { identity }\end{array}$ & $\begin{array}{l}\text { Cult. } \\
\text { method }\end{array}$ \\
\hline priv & & Eimeria maxima (A) $(\mathrm{m})$ & E h p & SAR - Alveolata - Apicomplexa - Coccidia & Guanine & + \\
\hline priv & & Psychodiella sergenti & E h p & SAR - Alveolata - Apicomplexa - Gregarinasina & Guanine & - \\
\hline NCMA & CCMP3155 & Vitrella brassicaformis & $\mathrm{Ma}$ & SAR - Alveolata - Chrompodellids - Vitrellaceae & Guanine & - \\
\hline ns & & Coleps sp. & $\mathrm{Fh}$ & SAR - Alveolata - Ciliophora - Prostomatea & Starch & - \\
\hline ns & & Cyclidium sp. & $\mathrm{Fh}$ & SAR - Alveolata - Ciliophora - Oligohymenophorea & Guanine & - \\
\hline ns & & Euplotes sp. & $\mathrm{Fh}$ & SAR - Alveolata - Ciliophora - Spirotrichea & Guanine & - \\
\hline ns & & Euplotes sp. & $\mathrm{Mh}$ & SAR - Alveolata - Ciliophora - Spirotrichea & Guanine & - \\
\hline ns & & Halteria sp. & $\mathrm{Fh}$ & SAR - Alveolata - Ciliophora - Spirotrichea & Guanine & - \\
\hline ns & & Glaucoma sp. & $\mathrm{Fh}$ & SAR - Alveolata - Ciliophora - Oligohymenophorea & Guanine & - \\
\hline ns & & Hypotrichia gen. sp. & $\mathrm{Mh}$ & SAR - Alveolata - Ciliophora - Spirotrichea & Guanine & - \\
\hline ns & & Lembadion sp. & $\mathrm{Mh}$ & SAR - Alveolata - Ciliophora - Oligohymenophorea & Guanine & - \\
\hline ns & & Oxytricha gen. sp. & $\mathrm{Fh}$ & SAR - Alveolata - Ciliophora - Spirotrichea & Guanine & - \\
\hline ns & & Paramecium sp. (C) (m) & $\mathrm{Fh}$ & SAR - Alveolata - Ciliophora - Oligohymenophorea & Guanine & + \\
\hline ns & & Scuticulociliatia gen. sp. & $\mathrm{Mh}$ & SAR - Alveolata - Ciliophora - Oligohymenophorea & Guanine & - \\
\hline ns & & Vorticella sp. & $\mathrm{Fh}$ & SAR - Alveolata - Ciliophora - Oligohymenophorea & - & - \\
\hline priv & & Borghiella sp. & $\mathrm{Fa}$ & SAR - Alveolata - Dinoglagellata - Suessiales & Guanine & - \\
\hline NCMA & CCMP449 & Heterocapsa triquetra & $\mathrm{Ma}$ & SAR - Alveolata - Dinoglagellata - Peridiniales & Guanine & $f / 2$ \\
\hline priv & & Glenodinium foliaceum (B) & $\mathrm{Ma}$ & SAR - Alveolata - Dinoglagellata - Peridiniales & Guanine & - \\
\hline ns & & Gymnodinium sp. & $\mathrm{Fa}$ & SAR - Alveolata - Dinoglagellata - Gymnodiniales & Guanine & - \\
\hline ns & & Peridinium sp. & $\mathrm{Fa}$ & SAR - Alveolata - Dinoglagellata - Peridiniales & Guanine & - \\
\hline priv & & Sphaerodinium sp. & $\mathrm{Fa}$ & SAR - Alveolata - Dinoglagellata - Suessiales & Guanine & - \\
\hline priv & 370 & Symbiodinium microadriaticum $(\mathrm{m})$ & $\mathrm{Ma}$ & SAR - Alveolata - Dinoglagellata - Suessiales & Guanine & $f / 2$ \\
\hline NCMA & CCMP829 & Symbiodinium tridacnidorum & $\mathrm{Ma}$ & SAR - Alveolata - Dinoglagellata - Suessiales & Guanine & $f / 2$ \\
\hline ns & & $\begin{array}{l}\text { Symbiodinium sp. } \\
\text { isolated from Capnella imbricata }\end{array}$ & $\mathrm{Ma}$ & SAR - Alveolata - Dinoglagellata - Suessiales & Guanine & $f / 2$ \\
\hline CAUP & J95 & Achnanthidium sp. & $\mathrm{Fa}$ & SAR - Stramenopiles - Ochrophyta - Bacillariophyceae & Calcite & - \\
\hline ns & & Asterionella formosa & $\mathrm{Fa}$ & SAR - Stramenopiles - Ochrophyta - Bacillariophyceae & - & - \\
\hline ns & & Aulacoseira sp. & $\mathrm{Fa}$ & SAR - Stramenopiles - Ochrophyta - Bacillariophyceae & - & - \\
\hline ns & & Encyonema sp. & $\mathrm{F} \mathrm{a}$ & SAR - Stramenopiles - Ochrophyta - Bacillariophyceae & Uric acid & - \\
\hline ns & & Entomoneis ornata & $\mathrm{M} \mathrm{a}$ & SAR - Stramenopiles - Ochrophyta - Bacillariophyceae & - & - \\
\hline ns & & Fragilaria sp. & $\mathrm{Fa}$ & SAR - Stramenopiles - Ochrophyta - Bacillariophyceae & Uric acid & - \\
\hline ns & & Frustulia sp. & $\mathrm{Fa}$ & SAR - Stramenopiles - Ochrophyta - Bacillariophyceae & - & - \\
\hline ns & & Gomphonema truncatum & $\mathrm{Fa}$ & SAR - Stramenopiles - Ochrophyta - Bacillariophyceae & - & - \\
\hline
\end{tabular}




\begin{tabular}{|c|c|c|c|c|c|c|}
\hline $\begin{array}{l}\text { Cult. } \\
\text { coll. }\end{array}$ & Code & Species & $\begin{array}{l}\text { Habitat } \\
\text {-trophy }\end{array}$ & Taxonomic classification & $\begin{array}{l}\text { Raman } \\
\text { identity }\end{array}$ & $\begin{array}{l}\text { Cult. } \\
\text { method }\end{array}$ \\
\hline ns & & Licmophora sp. & $\mathrm{M} \mathrm{a}$ & SAR - Stramenopiles - Ochrophyta - Bacillariophyceae & Calcite & - \\
\hline ns & & Melosira sp. & $\mathrm{Fa}$ & SAR - Stramenopiles - Ochrophyta - Bacillariophyceae & - & - \\
\hline ns & & Navicula cryptocephala & $\mathrm{Fa}$ & SAR - Stramenopiles - Ochrophyta - Bacillariophyceae & Uric acid & - \\
\hline ns & & Navicula sp. & $\mathrm{Fa}$ & SAR - Stramenopiles - Ochrophyta - Bacillariophyceae & $\begin{array}{l}\text { Uric acid } \\
\text { Xanthine }\end{array}$ & - \\
\hline ns & & $\begin{array}{l}\text { Naviculaceae gen. sp., Seminavis- } \\
\text { like }\end{array}$ & $\mathrm{Ma}$ & SAR - Stramenopiles - Ochrophyta - Bacillariophyceae & Guanine & - \\
\hline ns & & Paralia sulcata & $\mathrm{Ma}$ & SAR - Stramenopiles - Ochrophyta - Bacillariophyceae & Calcite & - \\
\hline CCAP & 1052/1B & Phaeodactylum tricornutum (m) & $\mathrm{Ma}$ & SAR - Stramenopiles - Ochrophyta - Bacillariophyceae & Calcite & $f / 2$ \\
\hline ns & & Pinnularia viridis & $\mathrm{Fa}$ & SAR - Stramenopiles - Ochrophyta - Bacillariophyceae & - & - \\
\hline ns & & Pleurosigma sp. & $\mathrm{Ma}$ & SAR - Stramenopiles - Ochrophyta - Bacillariophyceae & Uric acid & - \\
\hline ns & & Stauroneis phoenicenteron & $\mathrm{Fa}$ & SAR - Stramenopiles - Ochrophyta - Bacillariophyceae & - & - \\
\hline NCMA & CCMP1335 & Thalassiosira pseudonana (m) & $\mathrm{Ma}$ & SAR - Stramenopiles - Ochrophyta - Bacillariophyceae & - & $f / 2$ \\
\hline ns & & Spumella sp. & $\mathrm{Fa}$ & SAR - Stramenopiles - Ochrophyta - Synurophyceae & Guanine & - \\
\hline CAUP & B710 & Synura hibernica & $\mathrm{Fa}$ & SAR - Stramenopiles - Ochrophyta - Synurophyceae & Guanine & - \\
\hline CAUP & $\mathrm{H} 4302$ & Eustigmatos polyphem (b) & $\mathrm{Ta}$ & SAR - Stramenopiles - Ochrophyta - Eustigmatophyceae & Guanine & BBM \\
\hline NIES & 2146 & Nannochloropsis oculata (K) (b) (m) & $\mathrm{Ma}$ & SAR - Stramenopiles - Ochrophyta - Eustigmatophyceae & Guanine & $f / 2$ \\
\hline NIES & 2860 & Vacuoliviride crystalliferum ${ }^{\#}$ & Un a & SAR - Stramenopiles - Ochrophyta - Eustigmatophyceae & $\begin{array}{l}\text { Guanine } \\
\text { Chrysolaminarin }\end{array}$ & BBM \\
\hline ns & & Cystoseira sp. & $\mathrm{Ma}$ & SAR - Stramenopiles - Ochrophyta - Phaeophyceae & Calcite & - \\
\hline ns & & Dictyota dichotoma & $\mathrm{Ma}$ & SAR - Stramenopiles - Ochrophyta - Phaeophyceae & Calcite & - \\
\hline ns & & Padina pavonica & $\mathrm{Ma}$ & SAR - Stramenopiles - Ochrophyta - Phaeophyceae & Calcite & - \\
\hline ns & & Actinophrys sp. & $\mathrm{Fa}$ & SAR - Stramenopiles - Ochrophyta - Actinophryidae & Guanine & - \\
\hline ns & & Gonyostomum sp. & $\mathrm{Fa}$ & SAR - Stramenopiles - Ochrophyta - Raphidophyceae & Guanine & - \\
\hline CAUP & D301 & Botrydiopsis intercedens & $\mathrm{Fa}$ & SAR - Stramenopiles - Ochrophyta - Xanthophyceae & Guanine & BBM \\
\hline UTEX & B 2999 & Heterococcus sp. & $\mathrm{Fa}$ & SAR - Stramenopiles - Ochrophyta - Xanthophyceae & - & BBM \\
\hline ns & & Ophiocytium sp. & $\mathrm{Fa}$ & SAR - Stramenopiles - Ochrophyta - Xanthophyceae & Guanine & - \\
\hline CCALA & 517 & Tribonema aequale (F) (m) & $\mathrm{Fa}$ & SAR - Stramenopiles - Ochrophyta - Xanthophyceae & Guanine & BBM \\
\hline ns & & Xanthonema sp. & $\mathrm{Fa}$ & SAR - Stramenopiles - Ochrophyta - Xanthophyceae & Guanine & - \\
\hline DBM & $\mathrm{CO} 3 \mathrm{I}$ & Schizochytrium sp. (b) (m) & $\mathrm{M} \mathrm{h}$ & SAR - Stramenopiles - Labyrinthulomycota & Guanine & ASW-L \\
\hline priv & & Cafeteria roenbergensis & $\mathrm{M} \mathrm{h}$ & SAR - Stramenopiles - Bicosoecida & - & $f / 2^{*}$ \\
\hline priv & & Cafileria marina & $\mathrm{Mh}$ & SAR - Stramenopiles - Bicosoecida & Guanine & $f / 2^{*}$ \\
\hline CCF & 3762 & Phytophthora cactorum & E h & SAR - Stramenopiles - Oomycota & - & PDA \\
\hline
\end{tabular}




\begin{tabular}{|c|c|c|c|c|c|c|}
\hline $\begin{array}{l}\text { Cult. } \\
\text { coll. }\end{array}$ & Code & Species & $\begin{array}{l}\text { Habitat } \\
\text {-trophy }\end{array}$ & Taxonomic classification & $\begin{array}{l}\text { Raman } \\
\text { identity }\end{array}$ & $\begin{array}{l}\text { Cult. } \\
\text { method }\end{array}$ \\
\hline CCF & 4738 & Phytophthora rosacearum-like & E h & SAR - Stramenopiles - Oomycota & - & PDA \\
\hline priv & Ther & $\begin{array}{l}\text { Blastocystis sp. } \\
\text { isolated from Testudo hermanni }\end{array}$ & $\mathrm{E} \mathrm{h}$ & SAR - Stramenopiles - Opalinata - Blastocystae & - & TYM \\
\hline NCMA & CCMP2755 & Bigelowiella natans (L) (m) & $\mathrm{Ma}$ & SAR - Rhizaria - Cercozoa-Chlorarachnea & Guanine & $f / 2$ \\
\hline priv & LITO & Cercomonadida gen. sp. & $\mathrm{Fh}$ & SAR - Rhizaria - Cercozoa - Paracercomonadida & Guanine & Fw \\
\hline ns & & Viridiraptor invadens & $\mathrm{Fh}$ & SAR - Rhizaria - Cercozoa - Viridiraptoridae & Guanine & - \\
\hline ns & & Euglypha sp. & $\mathrm{Fh}$ & SAR - Rhizaria - Cercozoa - Euglyphida & Guanine & - \\
\hline ns & & Trinema sp. & $\mathrm{Fh}$ & SAR - Rhizaria - Cercozoa - Euglyphida & Guanine & - \\
\hline ns & & Marinomyxa marina & $\mathrm{M} \mathrm{h}$ & SAR - Rhizaria - Endomyxa - Phytomyxea & - & - \\
\hline ns & & Acantharea gen. sp. & $\mathrm{M} \mathrm{h}$ & SAR - Rhizaria - Retaria - Acantharea & Celestite & $\dagger$ \\
\hline ns & & Globigerinidae gen. sp. & $\mathrm{M} \mathrm{h}$ & SAR - Rhizaria - Retaria - Foramanifera & - & + \\
\hline ns & & Globorotaliidae gen. sp. & $\mathrm{M} \mathrm{h}$ & SAR - Rhizaria - Retaria - Foramanifera & Calcite & + \\
\hline priv & LIS-Tel & Telonema sp. & $\mathrm{Mh}$ & Telonemida & Guanine & $f / 2 B^{*}$ \\
\hline NCMA & CCMP371 & Emiliania huxleyi (m) & $\mathrm{Ma}$ & Haptista - Haptophyta & Guanine & $\mathrm{f} / 2^{*}$ \\
\hline $\mathrm{RCC}$ & RCC1350 & Isochrysis sp. (M) (b) & $\mathrm{Ma}$ & Haptista - Haptophyta & $\begin{array}{l}\text { Xanthine } \\
\text { Uric acid }\end{array}$ & $f / 2^{*}$ \\
\hline ns & & Raphidiophrys sp. & $\mathrm{Fh}$ & Haptista - Centroplasthelida & Guanine & - \\
\hline CAUP & F105 & Cryptomonas sp. (O) & $\mathrm{Fa}$ & Cryptista - Cryptophyta & Uric acid & BBM \\
\hline ns & & Chroomonas sp. & $\mathrm{Fa}$ & Cryptista - Cryptophyta & $\begin{array}{c}\text { Uric acid } \\
\text { Guanine } \\
\text { monohydrate }\end{array}$ & - \\
\hline CAUP & 0101 & Glaucocystis nostochinearum & $\mathrm{Fa}$ & Archaeplastida - Glaucophyta & Xanthine & BBM \\
\hline ns & & Asparagopsis taxiformis & $\mathrm{Ma}$ & Archaeplastida - Rhodophyta - Florideophyceae & Calcite & - \\
\hline CAUP & L201 & Asterocytis ramosa & $\mathrm{Ma}$ & Archaeplastida - Rhodophyta - Bangiophyceae & $\begin{array}{l}\text { Carotenoids, } \\
\text { lipids }\end{array}$ & $f / 2$ \\
\hline CCALA & 971 & Audouinella sp. & $\mathrm{Ma}$ & Archaeplastida - Rhodophyta - Florideophyceae & - & $f / 2$ \\
\hline ns & & Ceramium sp. & $\mathrm{Ma}$ & Archaeplastida - Rhodophyta - Florideophyceae & Calcite & - \\
\hline ns & & Hildebrandia rivularis & $\mathrm{Ma}$ & Archaeplastida - Rhodophyta - Florideophyceae & - & - \\
\hline CCALA & 416 & Porphyridium purpureum (b) & $\mathrm{Ma}$ & Archaeplastida - Rhodophyta - Porphyridiophyceae & - & $f / 2$ \\
\hline CCALA & 925 & Rhodella violacea & $\mathrm{Ma}$ & Archaeplastida - Rhodophyta - Rhodellophyceae & Lipids & $f / 2$ \\
\hline ns & & Asterococcus sp. & $\mathrm{Fa}$ & Archaeplastida - Viridiplantae - Chlorophyta - Chlorophyceae & Guanine & - \\
\hline ns & & Bulbochaete sp. & $\mathrm{Fa}$ & Archaeplastida - Viridiplantae - Chlorophyta - Chlorophyceae & Guanine & - \\
\hline CRC & CC-1690 & Chlamydomonas reinhardtii ${ }^{\#}(\mathrm{~m})$ & $\mathrm{Fa}$ & Archaeplastida - Viridiplantae - Chlorophyta - Chlorophyceae & Guanine & BBM \\
\hline CAUP & G224 & Chlamydomonas geitleri & $\mathrm{Fa}$ & Archaeplastida - Viridiplantae - Chlorophyta - Chlorophyceae & Guanine & BBM \\
\hline
\end{tabular}




\begin{tabular}{|c|c|c|c|c|c|c|}
\hline $\begin{array}{l}\text { Cult. } \\
\text { coll. }\end{array}$ & Code & Species & $\begin{array}{l}\text { Habitat } \\
\text {-trophy }\end{array}$ & Taxonomic classification & $\begin{array}{l}\text { Raman } \\
\text { identity }\end{array}$ & $\begin{array}{l}\text { Cult. } \\
\text { method }\end{array}$ \\
\hline ns & & Chlamydomonas sp. & $\mathrm{Fa}$ & Archaeplastida - Viridiplantae - Chlorophyta - Chlorophyceae & Guanine & - \\
\hline priv & AMAZONIE & Chlamydomonadales gen. sp. & $\mathrm{Fh}$ & Archaeplastida - Viridiplantae - Chlorophyta - Chlorophyceae & Guanine & Fw \\
\hline priv & KBEL1C & Polytoma sp. & $\mathrm{Fh}$ & Archaeplastida - Viridiplantae - Chlorophyta - Chlorophyceae & Guanine & Fw \\
\hline CAUP & H 6902 & Chlorochytrium lemnae & $E-F a$ & Archaeplastida - Viridiplantae - Chlorophyta - Chlorophyceae & Guanine & BBM \\
\hline priv & & Chloromonas arctica & $\mathrm{Sa}$ & Archaeplastida - Viridiplantae - Chlorophyta - Chlorophyceae & Guanine & BBM \\
\hline ns & & Desmodesmus quadricauda (m) & $\mathrm{Fa}$ & Archaeplastida - Viridiplantae - Chlorophyta - Chlorophyceae & Guanine & - \\
\hline ns & & Desmodesmus sp. & $\mathrm{Fa}$ & Archaeplastida - Viridiplantae - Chlorophyta - Chlorophyceae & Guanine & - \\
\hline ns & & Eudorina sp. & $\mathrm{Fa}$ & Archaeplastida - Viridiplantae - Chlorophyta - Chlorophyceae & Guanine & - \\
\hline ns & & Gleocystis sp. & $\mathrm{Fa}$ & Archaeplastida - Viridiplantae - Chlorophyta - Chlorophyceae & Guanine & - \\
\hline ns & & Microspora sp. & $\mathrm{Fa}$ & Archaeplastida - Viridiplantae - Chlorophyta - Chlorophyceae & Guanine & - \\
\hline ns & & Monactinus simplex & $\mathrm{Fa}$ & Archaeplastida - Viridiplantae - Chlorophyta - Chlorophyceae & Guanine & - \\
\hline CAUP & H2908 & Monoraphidium contortum (b) & $\mathrm{Fa}$ & Archaeplastida - Viridiplantae - Chlorophyta - Chlorophyceae & Guanine & BBM \\
\hline ns & & Oedogonium sp. & $\mathrm{Fa}$ & Archaeplastida - Viridiplantae - Chlorophyta - Chlorophyceae & Guanine & - \\
\hline ns & & Pediastrum boryanum & $\mathrm{Fa}$ & Archaeplastida - Viridiplantae - Chlorophyta - Chlorophyceae & Guanine & - \\
\hline CAUP & $\mathrm{H} 2308$ & Pediastrum duplex (I) & $\mathrm{Fa}$ & Archaeplastida - Viridiplantae - Chlorophyta - Chlorophyceae & Guanine & BBM \\
\hline ns & & Tetraedron sp. & $\mathrm{Fa}$ & Archaeplastida - Viridiplantae - Chlorophyta - Chlorophyceae & Guanine & - \\
\hline ns & & Tetraspora sp. & $\mathrm{Fa}$ & Archaeplastida - Viridiplantae - Chlorophyta - Chlorophyceae & Guanine & - \\
\hline CAUP & H 1917 & Chlorella vulgaris (N) (b) (m) & $\mathrm{Fa}$ & Archaeplastida - Viridiplantae - Chlorophyta - Trebouxiophyceae & Xanthine & $\mathrm{BBM}^{*}$ \\
\hline CAUP & H 5107 & Coccomyxa elongata & $\mathrm{Ta}$ & Archaeplastida - Viridiplantae - Chlorophyta - Trebouxiophyceae & Guanine & BBM \\
\hline ns & & Dictyosphaerium sp. (b) & $\mathrm{Fa}$ & Archaeplastida - Viridiplantae - Chlorophyta - Trebouxiophyceae & Uric acid & - \\
\hline ns & & Keratococcus sp. & $\mathrm{Fa}$ & Archaeplastida - Viridiplantae - Chlorophyta - Trebouxiophyceae & Guanine & - \\
\hline ns & & Micractinium sp. & $\mathrm{Fa}$ & Archaeplastida - Viridiplantae - Chlorophyta - Trebouxiophyceae & Guanine & - \\
\hline CAUP & H8801 & Phyllosiphon arisari & $\mathrm{Fap}$ & Archaeplastida - Viridiplantae - Chlorophyta - Trebouxiophyceae & Lipids & BBM \\
\hline CAUP & H8605 & Symbiochloris tschermakiae & $\mathrm{E} / \mathrm{T} \mathrm{a}$ & Archaeplastida - Viridiplantae - Chlorophyta - Trebouxiophyceae & Lipids & BBM \\
\hline ns & & Anadyomene sp. & $\mathrm{M} \mathrm{a}$ & Archaeplastida - Viridiplantae - Chlorophyta - Ulvophyceae & Calcite & - \\
\hline ns & & Cladophora sp. & $\mathrm{Fa}$ & Archaeplastida - Viridiplantae - Chlorophyta - Ulvophyceae & Guanine & - \\
\hline ns & & Cladophorasp. & $\mathrm{Ma}$ & Archaeplastida - Viridiplantae - Chlorophyta - Ulvophyceae & Calcite & - \\
\hline ns & & Enteromorpha sp. & $\mathrm{Ma}$ & Archaeplastida - Viridiplantae - Chlorophyta - Ulvophyceae & Calcite & - \\
\hline CAUP & H5301 & Scotinosphaera gibberosa & $\mathrm{F} / \mathrm{T} \mathrm{a}$ & Archaeplastida - Viridiplantae - Chlorophyta - Ulvophyceae & $\begin{array}{l}\text { Carotenoids, } \\
\text { lipids }\end{array}$ & BBM \\
\hline CAUP & J1601 & Trentepohlia sp. & $\mathrm{T}$ a & Archaeplastida - Viridiplantae - Chlorophyta - Ulvophyceae & $\begin{array}{l}\text { Carotenoids, } \\
\text { lipids }\end{array}$ & BBM \\
\hline
\end{tabular}




\begin{tabular}{|c|c|c|c|c|c|c|}
\hline $\begin{array}{l}\text { Cult. } \\
\text { coll. }\end{array}$ & Code & Species & $\begin{array}{l}\text { Habitat } \\
\text {-trophy }\end{array}$ & Taxonomic classification & $\begin{array}{l}\text { Raman } \\
\text { identity }\end{array}$ & $\begin{array}{l}\text { Cult. } \\
\text { method }\end{array}$ \\
\hline CAUP & M101 & Prasinocladus ascus & $\mathrm{Ma}$ & Archaeplastida - Viridiplantae - Chlorophyta - Chlorodendrophyceae & $\begin{array}{c}\text { Guanine } \\
\text { monohydrate } \\
\text { Uric acid }\end{array}$ & - \\
\hline CAUP & M201 & Tetraselmis subcordiformis (H) (b) & $\mathrm{Ma}$ & Archaeplastida - Viridiplantae - Chlorophyta - Chlorodendrophyceae & $\begin{array}{c}\text { Guanine } \\
\text { monohydrate } \\
\text { Uric acid }\end{array}$ & $f / 2$ \\
\hline RCC & RCC745 & Ostreococcus tauri (m) & $\mathrm{Ma}$ & Archaeplastida - Viridiplantae - Chlorophyta - Mamiellophyceae & - & $f / 2$ \\
\hline ns & & Nephroselmis sp. & $\mathrm{Ma}$ & Archaeplastida - Viridiplantae - Chlorophyta - Nephrophyceae & Guanine & - \\
\hline ns & & Pyramimonas sp. & $\mathrm{Ma}$ & Archaeplastida - Viridiplantae - Chlorophyta - Pyramimonadophyceae & Guanine & - \\
\hline NIES & 995 & Mesostigma viride & $\mathrm{Fa}$ & Archaeplastida - Viridiplantae - Streptophyta - Mesostigmatophyta & - & - \\
\hline CAUP & H7601 & Chlorokybus atmophyticus & $\mathrm{Ta}$ & Archaeplastida - Viridiplantae - Streptophyta - Chlorokybophyceae & Guanine & BBM \\
\hline NIES & 2285 & Klebsormidium flaccidum ${ }^{\#}$ (P) & $\mathrm{Ta}$ & Archaeplastida - Viridiplantae - Streptophyta - Klebsormidiophyceae & Uric acid & BBM \\
\hline ns & & Coleochaete sp. & $\mathrm{Fa}$ & Archaeplastida - Viridiplantae - Streptophyta - Coleochaetophyceae & Calcium oxalate & - \\
\hline NIES & 1604 & Chara braunii & $\mathrm{Fa}$ & Archaeplastida - Viridiplantae - Streptophyta - Charophyceae & - & - \\
\hline CAUP & K 801 & Actinotaenium sp. & $\mathrm{Fa}$ & Archaeplastida - Viridiplantae - Streptophyta - Zygnematophyceae & Uric acid & BBM \\
\hline NIES & 67 & $\begin{array}{l}\text { Closterium peracerosum-strigosum- } \\
\text { littorale complex (m) }\end{array}$ & $\mathrm{Fa}$ & Archaeplastida - Viridiplantae - Streptophyta - Zygnematophyceae & $\begin{array}{l}\text { Baryte } \\
\text { Starch }\end{array}$ & BBM \\
\hline ns & & Cosmarium sp. & $\mathrm{Fa}$ & Archaeplastida - Viridiplantae - Streptophyta - Zygnematophyceae & $\begin{array}{c}\text { Uric acid } \\
\text { Baryte }\end{array}$ & - \\
\hline ns & & Cylindrocystis sp. & $\mathrm{Ta}$ & Archaeplastida - Viridiplantae - Streptophyta - Zygnematophyceae & Calcium oxalate & - \\
\hline ns & & Euastrum humerosum & $\mathrm{Fa}$ & Archaeplastida - Viridiplantae - Streptophyta - Zygnematophyceae & Uric acid & - \\
\hline CAUP & K101 & Mesotaenium caldariorum & $\mathrm{Fa}$ & Archaeplastida - Viridiplantae - Streptophyta - Zygnematophyceae & $\begin{array}{c}\text { Uric acid } \\
\text { Guanine } \\
\text { monohydrate }\end{array}$ & $\mathrm{BBM}^{*}$ \\
\hline ns & & Mougeotia sp. & $\mathrm{Fa}$ & Archaeplastida - Viridiplantae - Streptophyta - Zygnematophyceae & Uric acid & - \\
\hline ns & & Netrium sp. & $\mathrm{Fa}$ & Archaeplastida - Viridiplantae - Streptophyta - Zygnematophyceae & Uric acid & - \\
\hline NIES & 303 & Penium margaritaceum (Q) & $\mathrm{Fa}$ & Archaeplastida - Viridiplantae - Streptophyta - Zygnematophyceae & Uric acid & BBM \\
\hline ns & & Spirogyra sp. & $\mathrm{Fa}$ & Archaeplastida - Viridiplantae - Streptophyta - Zygnematophyceae & Baryte & - \\
\hline ns & & Staurastrum sp. & $\mathrm{Fa}$ & Archaeplastida - Viridiplantae - Streptophyta - Zygnematophyceae & Uric acid & - \\
\hline $\mathrm{ns}$ & & Tetmemorus sp. & $\mathrm{Fa}$ & Archaeplastida - Viridiplantae - Streptophyta - Zygnematophyceae & Uric acid & - \\
\hline ns & & Xanthidium sp. & $\mathrm{Fa}$ & Archaeplastida - Viridiplantae - Streptophyta - Zygnematophyceae & Uric acid & - \\
\hline ns & & Halophila stipulacea & $\mathrm{Ma}$ & Archaeplastida - Viridiplantae - Streptophyta - Embryophyta & $\begin{array}{c}\text { Calcium oxalate } \\
\text { monohydrate, } \\
\text { dihydrate }\end{array}$ & - \\
\hline
\end{tabular}




\begin{tabular}{|c|c|c|c|c|c|c|}
\hline $\begin{array}{l}\text { Cult. } \\
\text { coll. }\end{array}$ & Code & Species & $\begin{array}{l}\text { Habitat } \\
\text {-trophy }\end{array}$ & Taxonomic classification & $\begin{array}{l}\text { Raman } \\
\text { identity }\end{array}$ & $\begin{array}{l}\text { Cult. } \\
\text { method }\end{array}$ \\
\hline priv & BY-2 & Nicotiana tabacum & $\mathrm{Ta}$ & Archaeplastida - Viridiplantae - Streptophyta - Embryophyta & $\begin{array}{l}\text { Calcium oxalate } \\
\text { monohydrate }\end{array}$ & MS-1 \\
\hline priv & & Physcomitrela patens & Ta & Archaeplastida - Viridiplantae - Streptophyta - Embryophyta & $\begin{array}{l}\text { Calcium oxalate } \\
\text { monohydrate }\end{array}$ & MS-2 \\
\hline priv & BW2 & Hemimastix kukwesjijk & $\mathrm{Fh}$ & Hemimastigophora & Guanine & FwB2* \\
\hline ns & & Entosiphon sp. & $\mathrm{Fa}$ & Excavata - Discoba - Euglenozoa - Euglenida & Guanine & - \\
\hline ns & & Euglena sp. $(\mathrm{m})$ & $\mathrm{Fa}$ & Excavata - Discoba - Euglenozoa - Euglenida & Guanine & - \\
\hline NCMA & CCMP1594 & Eutreptiela gymnastica (D) (m) & $\mathrm{Ma}$ & Excavata - Discoba - Euglenozoa - Euglenida & Guanine & $f / 2$ \\
\hline ns & & Lepocynclis oxyuris & $\mathrm{Fa}$ & Excavata - Discoba - Euglenozoa - Euglenida & Guanine & - \\
\hline ns & & Monomorphina pyrum & $\mathrm{Fa}$ & Excavata - Discoba - Euglenozoa - Euglenida & Guanine & - \\
\hline ns & & Phacus sp. & $\mathrm{Fa}$ & Excavata - Discoba - Euglenozoa - Euglenida & Guanine & - \\
\hline priv & SVATBA & Rhabdomonas sp. & $\mathrm{Fh}$ & Excavata - Discoba - Euglenozoa - Euglenida & Guanine & Fw \\
\hline ns & & Trachelomonas sp. & $\mathrm{Fa}$ & Excavata - Discoba - Euglenozoa - Euglenida & Guanine & - \\
\hline priv & $\mathrm{RIOZ}$ & Free-living Kinetoplastea gen. sp. & $\mathrm{Fh}$ & Excavata - Discoba - Euglenozoa - Kinetoplastea & Guanine & Fw \\
\hline ns & & Bodo sp. & $\mathrm{Fh}$ & Excavata - Discoba - Euglenozoa - Kinetoplastea & Guanine & - \\
\hline priv & M09 & 'jaculum'gen. sp. & E h p & Excavata - Discoba - Euglenozoa - Kinetoplastea & - & $\mathrm{BHI}$ \\
\hline priv & & Trypanosoma mega & $\mathrm{Ehp}$ & Excavata - Discoba - Euglenozoa - Kinetoplastea & - & $\mathrm{BHI}$ \\
\hline priv & YPF 1621 & Namystynia karyoxenos & $\mathrm{Mh}$ & Excavata - Discoba - Euglenozoa - Diplonemea & Guanine & ASW-D \\
\hline priv & 1.7 clone & Rhynchopus sp. & $\mathrm{Mh}$ & Excavata - Discoba - Euglenozoa - Diplonemea & Guanine & ASW-D \\
\hline priv & DT1610 & Flectonema sp. & $\mathrm{Mh}$ & Excavata - Discoba - Euglenozoa - Diplonemea & $\begin{array}{c}\text { Guanine } \\
\text { monohydrate }\end{array}$ & ASW-D \\
\hline priv & RUM4AN & Psalteriomonas lanterna & $\mathrm{Fh}$ & Excavata - Discoba - Heterolobosea & Guanine & Fw \\
\hline priv & NEG-M & Naegleria gruberi $(\mathrm{E})(\mathrm{m})$ & $\mathrm{Fh}$ & Excavata - Discoba - Heterolobosea & Guanine & $\mathrm{M7}^{*}$ \\
\hline priv & BUSSPRAND & Velundella trypanoides & $\mathrm{M} \mathrm{h}$ & Excavata - Discoba - Jakobida & - & JM \\
\hline priv & AND & Stygiella agilis & $\mathrm{Mh}$ & Excavata - Discoba - Jakobida & - & $\mathrm{JM}$ \\
\hline priv & SPINDL2 & Gyromonas ambulans & $\mathrm{Fh}$ & Excavata - Metamonada - Fornicata & Guanine & $\mathrm{Fw}^{*}$ \\
\hline priv & TUN2 & Hexamita sp. & $\mathrm{Fh}$ & Excavata - Metamonada - Fornicata & Guanine & $\mathrm{Fw}^{*}$ \\
\hline priv & LITO & Trepomonas rotans & $\mathrm{Fh}$ & Excavata - Metamonada - Fornicata & Guanine & $\mathrm{Fw}^{*}$ \\
\hline priv & BREZ2C & Trepomonas sp. & $\mathrm{Fh}$ & Excavata - Metamonada - Fornicata & Guanine & $\mathrm{Fw}^{*}$ \\
\hline priv & CONGO & Trepomonas steinii & $\mathrm{Fh}$ & Excavata - Metamonada - Fornicata & Guanine & LB \\
\hline priv & Ncub & $\begin{array}{l}\text { Macrotrichomonoides sp. } \\
\text { isolated from Neotermes cubanus }\end{array}$ & E h & Excavata - Metamonada - Parabasalia & Guanine & - \\
\hline priv & 249 & Gefionella okellyi (J) & $\mathrm{Fh}$ & Malawimonadida & Guanine & $f / 2 B^{*}$ \\
\hline ATCC & HM-1:IMSS & Entamoeba histolytica & E h & Amoebozoa - Evosea - Archamoebae & Lipids & TYI* \\
\hline
\end{tabular}




\begin{tabular}{|c|c|c|c|c|c|c|}
\hline $\begin{array}{l}\text { Cult. } \\
\text { coll. }\end{array}$ & Code & Species & $\begin{array}{l}\text { Habitat } \\
\text {-trophy }\end{array}$ & Taxonomic classification & $\begin{array}{l}\text { Raman } \\
\text { identity }\end{array}$ & $\begin{array}{c}\text { Cult. } \\
\text { method }\end{array}$ \\
\hline ATCC & 30984 & Mastigamoeba balamuthi & $\mathrm{Fh}$ & Amoebozoa - Evosea - Archamoebae & $\begin{array}{l}\text { Xanthine } \\
\text { Lipids }\end{array}$ & PYGC* \\
\hline priv & GO7 & Mastigella eilhardi & $\mathrm{Fh}$ & Amoebozoa - Evosea - Archamoebae & Guanine & $\mathrm{Fw}^{*}$ \\
\hline ns & & Fuligo septica & $\mathrm{Th}$ & Amoebozoa - Evosea - Eumycetozoa & Guanine & - \\
\hline priv & & Physarum polycephalum & $\mathrm{Th}$ & Amoebozoa - Evosea - Eumycetozoa & Xanthine & OG * \\
\hline priv & Neff & Acanthamoeba castellanii & $\mathrm{Fh}$ & Amoebozoa - Discosea - Centramoebia & $\begin{array}{c}\text { Xanthine } \\
\text { Lipids }\end{array}$ & Neff* \\
\hline ns & & Mayorella sp. & $\mathrm{Fh}$ & Amoebozoa - Discosea - Flabellinia & Xanthine & - \\
\hline $\mathrm{ns}$ & & Mayorella sp. & $\mathrm{M} \mathrm{h}$ & Amoebozoa - Discosea - Flabellinia & Xanthine & - \\
\hline priv & WFP252 & Neoparamoeba sp. & $\mathrm{M} \mathrm{h}$ & Amoebozoa - Discosea - Flabellinia & - & MAM \\
\hline ns & & Thecamoeba sp. & $\mathrm{Fh}$ & Amoebozoa - Discosea - Flabellinia & Guanine & - \\
\hline ns & & Vannella sp. & $\mathrm{Fh}$ & Amoebozoa - Discosea - Flabellinia & Guanine & - \\
\hline ns & & Difflugia sp. & $\mathrm{Fh}$ & Amoebozoa - Tubulinea - Arcellinida & Guanine & - \\
\hline ns & & Rhizamoeba sp. & $\mathrm{Fh}$ & Amoebozoa - Tubulinea - Leptomyxida & - & - \\
\hline ns & & Saccamoeba sp. & $\mathrm{Fh}$ & Amoebozoa - Tubulinea - Hartmannellidae & Unidentified & - \\
\hline priv & MSEDG & Saccamoebasp. & $\mathrm{Fh}$ & Amoebozoa - Tubulinea - Hartmannellidae & Unidentified & FwAM* \\
\hline priv & $4391 / 1$ & Vermamoeba vermiformis & $\mathrm{Fh}$ & Amoebozoa - Tubulinea - Echinamoebida & - & FwAM* \\
\hline priv & LIS-Man & Mantamonas sp. & $\mathrm{M} \mathrm{h}$ & CRuMs - Mantamonadida & - & $f / 2 B *$ \\
\hline priv & FB10 & Subulatomonas sp. & $\mathrm{M} \mathrm{h}$ & Obazoa - Breviatea & - & LBM \\
\hline priv & $\mathrm{C} 3 \mathrm{C}$ & Choanoflagellata gen. sp. & $\mathrm{Hph}$ & Obazoa - Opisthokonta - Choanoflagellata & - & $\mathrm{HM}^{*} \mathrm{~V}^{*}$ \\
\hline ns & & Chaetonotidae gen. sp. & $\mathrm{Fh}$ & Obazoa - Opisthokonta - Metazoa - Gastrotricha & Guanine & - \\
\hline ns & & Nematoda gen. sp. & $\mathrm{F} / \mathrm{T} \mathrm{h}$ & Obazoa - Opisthokonta - Metazoa - Nematoda & Uric acid & - \\
\hline ns & & Oncorhynchus mykiss (scale) & $\mathrm{Fh}$ & Obazoa - Opisthokonta - Metazoa - Chordata & Guanine & - \\
\hline CCF & 2912 & Aspergillus nidulans & $\mathrm{F} / \mathrm{Th}$ & Obazoa - Opisthokonta - Fungi - Ascomycota - Eurotiales & - & MEA \\
\hline ATCC & SC5314 & Candida albicans & $\mathrm{F} / \mathrm{Th}$ & Obazoa - Opisthokonta - Fungi - Ascomycota - Saccharomycetales & Guanine & MEA \\
\hline $\begin{array}{l}\text { EUROS } \\
\text { CARF }\end{array}$ & BY4741 & Saccharomyces cerevisiae & $\mathrm{Th}$ & Obazoa - Opisthokonta - Fungi - Ascomycota - Saccharomycetales & - & YPAD \\
\hline CCF & 3485 & Neurospora sitophila & $\mathrm{F} / \mathrm{Th}$ & Obazoa - Opisthokonta - Fungi - Ascomycota - Sordariales & - & MEA \\
\hline CCVC & CQ1 & Nosema bombycis & E h p & Obazoa - Opisthokonta - Opisthosporidia - Microsporidia & - & - \\
\hline ns & & Woronichinia naegeliana & $\mathrm{Fa}$ & Eubacteria - Cyanobacteria & Aerotopes & - \\
\hline ns & & Oscillatoria sp. & $\mathrm{Fa}$ & Eubacteria - Cyanobacteria & $\begin{array}{l}\text { Carotenoids, } \\
\text { lipids }\end{array}$ & - \\
\hline priv & & Bacillus subtilis & Th & Eubacteria - Firmicutes & - & \\
\hline
\end{tabular}


Table S2. Overview of the Concentrative nucleoside transporter (CNT) distribution among eukaryotes based on HMM search in the database of 57 eukaryotic genomes.

\begin{tabular}{|l|c|}
\hline Taxonomic rank & Presence of CNT \\
\hline Amoebozoa & yes \\
\hline Holomycota & yes \\
\hline Chrompodellids & yes \\
\hline Streptophyta & no \\
\hline Rhizaria & yes \\
\hline Stramenopiles & no \\
\hline Holozoa & yes \\
\hline Chlorophyta & no \\
\hline Haptophyta & yes \\
\hline Cryptophyceae & yes \\
\hline Apicomplexa & no \\
\hline Rhodophyta & no \\
\hline Euglenozoa & no \\
\hline Metamonada & no \\
\hline Ciliophora & no \\
\hline Heterolobosea & no \\
\hline Perkinsidae & no \\
\hline Apusomonadida & no \\
\hline
\end{tabular}


Table S3. Overview of the Equilibrative nucleoside transporter (ENT) distribution among eukaryotes based on HMM search in the database of 57 eukaryotic genomes.

\begin{tabular}{|l|c|}
\hline Taxonomic rank & Presence of ENT \\
\hline Amoebozoa: & yes \\
\hline Holomycota: & yes \\
\hline Chrompodellids & yes \\
\hline Streptophyta & yes \\
\hline Rhizaria & yes \\
\hline Stramenopiles & yes \\
\hline Holozoa & yes \\
\hline Chlorophyta & yes \\
\hline Haptophyta & yes \\
\hline Cryptophyceae & yes \\
\hline Apicomplexa & yes \\
\hline Rhodophyta & yes \\
\hline Euglenozoa & yes \\
\hline Metamonada & yes \\
\hline Ciliophora & yes \\
\hline Heterolobosea & yes \\
\hline Perkinsidae & yes \\
\hline Apusomonadida & yes \\
\hline
\end{tabular}




\section{Movie S1.}

Polarized light microscopy of crystalline inclusions in SAR.

https://youtu.be/cMkMJthq5KQ

\section{Movie S2.}

Polarized light microscopy of crystalline inclusions in Haptista, Cryptista and Telonemia.

https://youtu.be/Z30CDbWqOhc

\section{Movie S3.}

Polarized light microscopy of crystalline inclusions in Archaeplastida.

https://youtu.be/2ZXfOdpsJcU

\section{Movie S4.}

Polarized light microscopy of crystalline inclusions in Amoebozoa.

https://youtu.be/DUbA5e_1_BE

\section{Movie S5.}

Polarized light microscopy of crystalline inclusions in Opisthokonta.

https://youtu.be/kyEzbo-IbiM

\section{Movie S6.}

Polarized light microscopy of crystalline inclusions in Excavata.

https://youtu.be/XWzNBLmE01A

\section{Movie S7.}

Polarized light microscopy of crystalline inclusions in Hemimastigophora.

https://youtu.be/gnUZhZRRfcw

\section{Movie S8.}

Polarized light microscopy of crystalline inclusions in Prokaryota - Cyanobacteria.

https://youtu.be/8yGo161rdJU

Correspondence and requests for materials should be addressed to:

jana.pilatova@natur.cuni.cz 